\title{
Şanlıurfa İlinde Tüketilen Kaçak Çaylarda LC-MS ve GC-MS ile Pestisit Analizi
}

\author{
Kasım TAKIM ${ }^{1}$ (D), Mehmet Emin AYDEMIR ${ }^{2}$ iD \\ ${ }^{1}$ Harran Üniversitesi Veteriner Fakültesi, Veteriner Hekimliği Temel Bilimleri Bölümü, 63200 Eyyübiye, Şanlıurfa, ${ }^{2}$ Harran Üniversitesi \\ Veteriner Fakültesi, Veteriner Gıda Hijyeni ve Teknolojisi Bölümü, 63200 Eyyübiye, Şanlıurfa \\ 凹:kasimtakim@hotmail.com
}

\section{ÖZET}

Bu çalışmada; Türkiye'nin Şanlıurfa ilinde halk tarafından sevilerek tüketilen; İran ve Sri Lanka menşeli Seylan çaylarında pestisit ilaç kalıntısı analizleri yapılmıştır. Çalışma kapsamında semt pazarlarında satılan markalı ve markasız kaçak çaylardan numuneler alınmış ve içerlerinde bulunabilecek 594 adet pestisit kalıntısı için, sıvı kromatografisi/çift kütle spektrofotometre (LCMS/MS) ve gaz kromatografisi/kütle spektrofotometre (GC-MS/MS) cihazlarıyla analiz edilmişlerdir. LS-MS/MS ve GC-MS/MS cihazlarından alınan sonuçlara göre çalışılan örneklerde herhangi bir pestisit kalıntısına rastlanmamıştır. Literatürde kaçak çayların pestisit içerdiği ile ilgili pek çok iddia yer almaktadır. Bu iddialar analiz sonuçlarına göre değil, tahminler üzerine kurulduğu için spekülasyon olmaktan öteye geçmemektedir. Yaptığımız çalışmadan elde edilen veriler, literatürdeki spekülasyonlarla çelişmektedir. $\mathrm{Bu}$ yüzden bölge genelini kapsayacak daha geniş çalışmalarla bu karmaşıklığın ortadan kaldırılması gerekmektedir. Bunun için GAP bölgesinin tamamından kaçak çay numunelerinin toplanması ve bu örneklerle ilgili analizlerin genişletilerek yapılması büyük bir önem arz etmektedir.

\section{Pesticide Analysis by LC-MS and GC-MS in Leaky Tea Consumed in Şanlıurfa}

\section{ABSTRACT}

The aim of this study was to evaluate the presence of pesticide residues in Iranian and Sri Lankan origin leaky teas consumed by public in Şanliurfa Province of Turkey. Within the scope of this study, the samples were taken from branded and brand-free illegal teas in the bazaar. These samples were analyzed in Luquid ChromotagrophyMass and Spectrophotometry Gas Chromotagrophy-Mass Spectrophotometry (LC-MS / MS and GC-MS / MS) to evaluate the presence of 594 pesticide residues. According to the results of chromatograms, no pesticide residues were found in the leaky teas samples studied. Although there are many claims in the literature about the presence of pesticides in illegal teas, these claims do not go beyond speculation because they are based on estimates, not on the results of analysis. However, the data obtained this study are contradictory in the literature only as speculation. Therefore, this complexity needs to be clarified through a more extensive work to be done across the region.Therefore, collecting leaky tea samples from the entire GAP region and expanding the analyzes of these samples are of great importance.
DOI:10.18016/ksudobil.402273

\section{Makale Tarihçesi}

Geliş Tarihi : 06.03.2018

Kabul Tarihi :16.05.2018

\section{Anahtar Kelimeler}

Çay,

Kaçak Çay,

Pestisit

\section{Araştırma Makalesi}

\section{Article History}

Received : 06.03.2018

Accepted : 16.05.2018

\section{Keywords}

Tea,

Leaky Tea,

Pesticide

\section{Research Article}

To cite: Takım K, Aydemir ME 2018. Şanlıurfa İlinde Tüketilen Kaçak Çaylarda LC-MS ve GC-MS ile Pestisit Analizi. KSÜ Tarım ve Doğa Derg 21(5): 650-664. DOI:10.18016/ksudobil.402273.

\section{GİRIŞ}

Çay (Camellia sinensis); çaygiller familyasından (Theaceae) tropikal ve astropikal iklimlerde yetişen bitkinin yapraklarından elde edilir. Dünya üzerinde ilk olarak Çin ve Hindistan'da yetiştirilmeye başlanmıştır. Anavatanı Assam (Hindistan'ın Çin'e bakan iç tarafları)'dır (Üstün ve Demirci, 2013). Çay bitkisinin yapraklarının ve tomurcuklarının değişik metotlarla işlenmesi sonucu elde edilen çay, dünyada sudan sonra en fazla sevilerek içilen ve içme 
alışkanlığı gittikçe artan 500 yıllık bir geçmişe sahip ekonomik değer taşıyan bir içecektir. Çay; kurutularak işlenmesi ve her mevsimde tüketilmesi gibi avantajlarından dolayı günlük bir içecek olmayı başarmıştır. Ayrıca uykuya karşı koymak, dinlenmek, ısınmak ya da serinlemek amacıyla keyif verici içecek olarak da insanlar tarafindan tüketilmektedir (Kurt ve Hacığlu, 2013).

Çay bitkisi dünya üzerinde, kuzey yarım kürede yaklaşık 42 enlem derecesinden, güney yarım kürede 27 enlem derecesine kadar olan kuşak üzerinde yetiştirilmektedir. Sri Lanka çay bitkisinin yaygın olarak yetiştirildiği ülkelerden biridir. Dünya çay ihracatında en önemli ülkeler arasında yaklaşık \% 25'lik pay ve toplam 1.365.966 dolarlık ihracat ile Sri Lanka, \% 21'lik pay ve 1.165 .137 dolar ihracat ile Kenya ve \% 12'lik pay ve 694.661 dolarlık ihracat ile Hindistan yer almaktadır (Kurt ve Hacıoğlu, 2013). Sri Lanka çay üretimi ve çeşitliliği konusunda oldukça etkin bir ülke olarak rol oynamaktadır. Öyle ki; ilk organik çay üretimi 1986 yllında Sri Lanka'da yapılmıştır (Gültekin, 2013). Türkiye ile birlikte 30'a yakın ülkede ekonomik düzeyde çay üretimi yapılmaktadır (Harman, 2014). Ülkemiz Çin, Hindistan, Kenya ve Sri Lanka'dan sonra dünyadaki beşinci en büyük siyah çay üreticisidir (FAO, 2013). Çay bitkisi yetiştirilen bu ülkelerin çoğu ekvatoral ve ekvatora yakın bölgelerde bulunmaktadır. Çayda bakteriyel ve mantar hastalıklarının yanı sira 160 farklı böceğin olduğu tespit edildiği bu bölgelerde kış mevsiminin yok kabul edilmesinin yanında iklimin nemli olması, bitki hastalık ve zararlıları ile kimyasal bir mücadeleyi gerekli kılmaktadır (Anonim, 2018-A). Bunun aksine Doğu Karadeniz bölgesinde yetişen çaylar, coğrafi ve ekolojik şartlar nedeniyle kış aylarında az da olsa kar altında kalmaktadır. Kar altında kalan çay bitkisinde bakteriyel ve mantar hastalıkları ve böcek bulunma riski azalmaktadır. Bu sebepten dolayı bu yörelerde yetişen çay bitkisi için kimyasal ilaçlar ile mücadeleye gerek kalmamaktadır (Zenginbal, 2013).

Ülkemizde tüketilen çaylar; Doğu Karadenizde yetişen çaylar ve ülkemize kaçak yollar ile gelen, özellikle güneydoğuda sıklıkla tüketilen kaçak çay diye adlandırılan çay olmak üzere ikiye ayrılır. Son dönemlerde Doğu ve Güneydoğu Anadolu Bölgesindeki sınır kapılarından çeşitli illegal yöntemler ile ülkemize getirilen İran meşeli kaçak çaylar, Türkiye'deki çay sektörünü olumsuz etkilemektedir (Rize Ticaret Borsası, 2014). Çayda uygulanan ve \%145'leri bulan yüksek vergiler, kaçak çay ithalatının artmasına sebep olmaktadır. Doğu ve Güneydoğu Anadolu Bölgesinde çay kaçakçılığı ticaret haline gelmiştir. Yapılan tüm uyarı ve önlemlere rağmen bu ticaret engellenememiştir, hatta artarak devam etmektedir (Er, 2013).

Bitkilerde de kullanılan kimyasalların (pestisitler) vücuda alınması sonucu değişik organlarda toksik etki oluşturabilirler. Özellikle İnsanlarda akut ve kronik zehirlenmeler, oksidatif stres, mutajenik ve karsinojenik etkiler gibi ciddi sağllk problemlerine yol açabilmektedirler (Eren ve ark., 2006). Pestisitlerin sebep olduğu akut etkiler, irritasyondan dermatite ve hatta sistemik emilime bağll olarak ölüme kadar değişebilmektedir (Soyöz ve Özçelik, 2003). Kronik etkiler; Non Hodgkin lenfoma, lösemi, multiple myeloma, karaciğer kanseri, doğum defektleri, nörotoksisite (nöropatiye, demiyelinizasyona) nörodavranışsal bozukluklar, nörofizyolojik değişiklikler, üreme ve fertilite (düşük, düşük doğum ağırlığı, toksemi ve postpartum kanama) üzerindeki etkiler olarak sıralanabilir (Şensoy ve ark., 2017).

Pestisitlerin halk sağlığı üzerinde bu kadar ciddi sağllk sorunlarına sebep olması, ülkemize İran ve Sri Lanka'dan kaçak yollar ile giren çaylarda da pestisit ilaçların kullanıldığının iddia edilmesi, kaçak çaylarda pestisit analizlerin yapılmasını oldukça önemli bir hale getirmektedir.

\section{MATERYAL ve METOT}

Şanlıurfa ili semt pazarlarında satılmakta olan seylan tipi kaçak çaylardan numuneler toplanıp analizin yapıldığı laboratuvara gönderilmiştir. Çay numuneleri aynı gün yapılacak LC-MS/MS analizi için hazırlanmıştır. $\mathrm{Bu}$ hazırlı şu şekilde gerçekleştirilmiştir: numuneden $5 \mathrm{~g}$ tartılıp üzerine 15 $\mathrm{mL}$ su ve $15 \mathrm{~mL}$ asetonitril ilave edilmiş, ve tüp 1 dakika boyunca çalkalanmıştır. Daha sonra QuEChERS (Quick, Easy, Cheap, Effective, Rugged and Safe) içerisindeki tuz eklenip 1 dakika daha tüp çalkalanmıştır. Bu işlemden sonra ekstrakt, 5 dakika boyunca 4000 rpm'de santrifüjlenmiş, sonrasında tüpte bulunan üst faz kısmından $1.5 \mathrm{~mL}$ alınıp $0.45^{\prime} \mathrm{lik}$ filtre ile filtrelenerek cihaza verilmiştir. GC-MS için ekstratın hazırlanması durumunda ise LC-MS/MS cihazı için hazırladığımız örnek hazırlama metodu aynen takip edilerek, santrifüj sonrası oluşan üst fazdan $8 \mathrm{~mL}$ alındı clean-up kit tüpüne ilave edilmiş ve 1 dakika boyunca tüp çalkalanmıştır. Daha sonra tüp 3 dakika boyunca 5000 rpm'de santrifüjlenip sıvı faz çekilmiş ve $0.45^{\prime}$ lik filtre ile filtrelenerek cihaza verilmiştir. Analizler Delta Laboratuvar Sist. Tezg. San. Tic. Şti, laboratuarında yaptırılmıştır. Analiz sonuçlarını teyit ettirmek için numune sayısı artırılarak, T.C. Gıda Tarım ve Hayvancılık Bakanlığı MSM Özel Gıda Kontrol Labaratuarı'nda ikinci bir analiz daha yaptırılmıştır. Analiz için, AOAC 2007.01 yöntemi kullanılmıştır (Lesueur ve ark., 2008). Bu metoda göre; $10 \mathrm{mg}$ homojenize edilmiş kaçak çay numunesi üzerine $10 \mathrm{~mL}$ asetonitrilin eklendi, ardından $100 \mu \mathrm{L}$ standart (ISTD) solüsyonu eklendi. Tüp kapatıldı ve 1 dakika boyunca elle iyice çalkalandı. Faz ayrımı ve pestisit bölümlenmesini sağlamak için bir tampon-tuz karışımı (4 gr magnezyum sülfat susuz kum $\left(\mathrm{MgSO}_{4}\right), 1$ gr sodyum 
klorür $(\mathrm{NaCl}), \quad 0.5 \mathrm{~g}$ disodyum hidrojen sitrat seskihidrat $\left(\mathrm{Cr}^{-} 6, \mathrm{H}_{6} \mathrm{Na}_{2} \mathrm{O}_{7} \cdot 1.5 \mathrm{H}_{2} \mathrm{O}\right)$ ve $1 \mathrm{gr}$ trisodyum sitrat dihidrat) eklenmiştir. Tüp kapatıldı, 1 dakika boyunca elle iyice çalkalandı ve 5 dakika 3500 rmp'de devirde santrifüjlendi. santrifüj tüpüne $15 \mathrm{mg}$ PSA ve $950 \mathrm{mg} \mathrm{MgSO}_{4}$ eklendi. 3 dakika 5000 rpm'de santrifüj edilip, 0.45 mikron filtreden geçirilip cihaza verildi. Birim olarak; her bir maddenin $\mathrm{kg}$ başına $\mathrm{mg}$ seviyesinde derişimi $(\mathrm{mg} / \mathrm{kg})$ baz alınmıştır. Bileşik analizleri, analiz cihazının sahip olduğu kütüphane veri tabanına göre değerlendirilmiştir.

Yapılan analizlerde iki farklı kromatografik teknik olan; LC-MS/MS ve GC-MS/MS yöntemleri kullanılmıştır. Bunun nedeni ise; analizi yapılan bileşiklerin çözünürlük farklılıklarından kaynaklanmaktadır. Polar olup suda çözünen bileşikler için LC-MS/MS yeterli olabilirken, apolar olup suda çözünemeyen bileşikler için GC-MS/MS gerekmektedir.

\section{Pestisit Kalıntılarının LC-MS/MS ile Tayini:}

LC-MS ile pestisit bileşiklerin analizi, ikili MS cihazı bağlanmış bir Nexera modeli Shimadzu HPLC kullanılarak yapılmıştır. Cihazın MS kütüphanesinde kayıtlı bileşiklerle karşılaştırılarak, numune içerisinde pestisit bileşikler belirlenmeye çalışılmıştır. Cihaza bağlı ekipmanlar ve çalışma şartları ise aşağıdaki şekilde ayarlanmıştır. Ekipmanları: LC20AD İki adet pompa, DGU-20A3R degaser, CTO10ASVP kolon firmn, SIL-20AC autosampler. Kolan Bilgileri: C-18 Intersil ODS-4 (3.0mm x $100 \mathrm{~mm}, 2 \mu \mathrm{m})$ analitik kolon Kolon sicaklığ̣: $400 \mathrm{C}$ Mobil faz: A (Su, \%0,1 Formik asit), B (Metanol, \%0,1 Formik asit). Akış $0.3 \mathrm{ml} / \mathrm{dk}$ ve enjeksiyon hacmi $2 \mu \mathrm{L}$.

\section{Pestisit Kalıntılarının GC-MS/MS ile Tayini:}

Pestisit bileşenlerin ayırımında, Shimadzu GC-2010; QP-2010 sistem; Shimadzu, Kyoto, Japonya DBFFAP kapiler kolon (60 m x 0,25 mm x 0,25 $\mu \mathrm{m}$; J\&W Scientific, Folsom, CA, A.B.D) kullanılmış ve cihazın enjeksiyon sicaklığ $250^{\circ} \mathrm{C}$, MS iyon kaynağ 1 ise $200^{\circ} \mathrm{C}^{\prime}$ ye ayarlanmıştır. Kütle spektrometresi ile 1 saniye aralıklarla 33-650 kütle/yük $(\mathrm{m} / \mathrm{z})$ arasında tarama yapılmış, seperasyon için sıcaklık programı $40^{\circ} \mathrm{C}$ ' den kademeli olarak $240^{\circ} \mathrm{C}$ ' ye çıkarılarak bu sıcaklıkta $6 \mathrm{dk}$ bekleyecek şekilde ayarlanmıştır. Taşıyıcı gaz olarak He kullanılarak kolon içerisinde $0.0166 \mathrm{~mL} / \mathrm{s}$ hızında akış sağlanmıştır. Piklerin tanımlanmasında MS kütüphanesinde bulunan Wiley 7 ve NIST 147 programları kullanılmıştır. Tanımlanan piklerin alıkonma indeksleri (retention index) $\mathrm{C}_{10^{-}}$ $\mathrm{C}_{26}$ n-alkan serisi (Dr. Ehrenstorfer GmbH, Ausburg, Almanya) kullanılarak belirlenmiş ve literatür verileriyle karşılaştırılmıştır.

\section{BULGULAR}

Bu çalışmada Çizelge 1'de görüldüğü gibi LC-MS/MS ve GC-MS ile analizi yapılan pestisit bileşiklerinin tümünde negatif sonuçlar çıkmıştır. Yine Şekil 1 ve Şeki 2'de görüldüğü gibi pestisit varlığını gösterecek bir pike rastlanmamış olup sadece ortam ve cihaz gürültüsüne bağlı piklere rastlanmıştır.

\section{TARTIŞMA ve SONUÇ}

Kaçak çay tüketimi Doğu ve Güney Doğu illerinde gün geçtikçe artmaktadır. Kaçak çay tüketiminin sağlık üzerinde birçok olumuz etkisi olduğu söylenilmektedir. Ancak bu güne kadar ülkemizde, kaçak çay ile ilgili ileri sürülen iddiaları doğrulayan ya da yanlış olduğunu gösteren bir bilimsel çalışma yapılmamıştır. Dolayısıyla da bu konudaki iddialar spekülasyon olmanın ötesine gitmemektedir. Bu tür spekülasyonlara örnek verecek olursak; Rize Ticaret Borsası'nın 2014 yılında yaptığı bir açıklamada: "İran'da çay üretimi yapıldığ sebebiyle çaylarda aşırı böceklenme meydana gelmektedir. Bu böceklenme ile mücadele edilmesi için pestisit ilaçlar kullanılmaktadır. Dolayısıyla İran çayında yüksek miktarda pestisit kalıntısı oluşmakta olduğu, bu çayları tüketen insanların sağlığı olumsuz yönde etkilendiği" belirtilmiştir (Rize Ticaret Borsası, 2014). Özcan ve arkadaşları yaptıkları bildiri sunumunda; "kaçak çayın ülkemize girişinin sadece çay arzını arttırmaya neden olmayıp, aynı zamanda kimyasal kalıntılar ve hormon içermesi bakımında halk sağlığını tehdit ettiğini” bildirmişlerdir (Özcan ve Yazıcıŏ̆lu, 2013). Yapılan bir çalışmada; İran menşeyli çayların üretim bakımından Türk çayına benzerlik gösterdiği fakat yüksek miktarda pestisit içerdiği, yapılan analizlerinde böcek bulunduğu ve hijyen bakımından sakıncalı olduğu ileri sürülmüştür (Harman, 2014). 2015 Yllında Diyarbakır ilinde yapılan doğal ve sağlıklı çay sempozyumunda ÇAYKUR genel müdürü; 'Dünyada en sağlıklı çayın ülkemizde Karadeniz bölgesinde üretildiğini, diğer ülkelerde yetiştirilen çayların kışın kar altında kalmadığından yüksek miktarda pestisit kalıntısı ve haşere içerdiğini, bu yüzden ülkemize kaçak yollar ile gelen çayların sağlıklı olmadığını" ifade etmiştir (Anonim 2018-C). Ulusal Çay Konseyinin yayımlamış olduğu İran Çayı raporunda: İran'da üretimi yapılan çaylarda böceklenmeyi önlemek için aşırı pestisit ilaçlar kullanıldığı, ilaçlanma sonrası ölen böceklerin çay bitkisinin yaprağına yapışı halde kaldığı, buna bağlı olarak İran çayında hem böcek kalıntıları hem de yüksek miktarda pestisit bulunduğu, sonuçta da kanserojen etkiye sahip bu kalıntıların ölüme varan birçok hastalığa davetiye çıkardığı" belirtilmiştir (Anonim 2018-B). 
Çizelge 1. Kaçak çayda LC-MS/MS ve GC-MS teknikleri ile analizi yapılan pestisit bileşiklerin limit değerleri ve analiz sonuçları.

\begin{tabular}{|c|c|c|c|c|}
\hline $\begin{array}{l}\text { Yapılan Analizler } \\
\text { Pestisit Kalıntıları }\end{array}$ & Sonuç & Ölçüm Limiti & Birim & $\begin{array}{l}\text { Analiz Metodu } \\
\text { QuEChERS, AOAC 2007.01 } \\
\text { (LC-MS/MS \& GC - MS, GC-MS/MS) }\end{array}$ \\
\hline 1-Naphthylacetamide (1-NAD) & Tespit Edilemedi & 0.010 & $\mathrm{mg} / \mathrm{kg}$ & \\
\hline $\mathrm{Q} 2$ & Tespit Edilemedi & 0.010 & $\mathrm{mg} / \mathrm{kg}$ & LC-MS/MS \\
\hline Acephate & Tespit Edilemedi & 0.010 & $\mathrm{mg} / \mathrm{kg}$ & LC-MS/MS \\
\hline Acequinocyl & Tespit Edilemedi & 0.010 & $\mathrm{mg} / \mathrm{kg}$ & LC-MS/MS \\
\hline Acetamiprid * & Tespit Edilemedi & 0.010 & $\mathrm{mg} / \mathrm{kg}$ & LC-MS/MS \\
\hline Acetochlor * & Tespit Edilemedi & 0.010 & $\mathrm{mg} / \mathrm{kg}$ & GC-MS \& GC-MS/MS \\
\hline Acibenzolar-S-Methyl & Tespit Edilemedi & 0.010 & $\mathrm{mg} / \mathrm{kg}$ & LC-MS/MS \\
\hline Aclonifen & Tespit Edilemedi & 0.010 & $\mathrm{mg} / \mathrm{kg}$ & GC-MS \& GC-MS/MS \\
\hline Acrinathrin * & Tespit Edilemedi & 0.010 & $\mathrm{mg} / \mathrm{kg}$ & GC-MS \& GC-MS/MS \\
\hline Alachlor * & Tespit Edilemedi & 0.010 & $\mathrm{mg} / \mathrm{kg}$ & GC-MS \& GC-MS/MS \\
\hline Aldicarb * & Tespit Edilemedi & 0.010 & $\mathrm{mg} / \mathrm{kg}$ & LC-MS/MS \\
\hline Aldicarb-Sulfone (Aldoxycarb) ${ }^{*}$ & Tespit Edilemedi & 0.010 & $\mathrm{mg} / \mathrm{kg}$ & LC-MS/MS \\
\hline Aldicarb-Sulfoxide * & Tespit Edilemedi & 0.010 & $\mathrm{mg} / \mathrm{kg}$ & LC-MS/MS \\
\hline Aldrin (HHDN) ${ }^{*}$ & Tespit Edilemedi & 0.010 & $\mathrm{mg} / \mathrm{kg}$ & GC-MS \& GC-MS/MS \\
\hline Allethrin & Tespit Edilemedi & 0.010 & $\mathrm{mg} / \mathrm{kg}$ & GC-MS \& GC-MS/MS \\
\hline alpha-Cypermethrin * & Tespit Edilemedi & 0.010 & $\mathrm{mg} / \mathrm{kg}$ & GC-MS \& GC-MS/MS \\
\hline Ametryn & Tespit Edilemedi & 0.010 & $\mathrm{mg} / \mathrm{kg}$ & LC-MS/MS \\
\hline Amidosulfuron * & Tespit Edilemedi & 0.010 & $\mathrm{mg} / \mathrm{kg}$ & LC-MS/MS \\
\hline Aminocarb & Tespit Edilemedi & 0.010 & $\mathrm{mg} / \mathrm{kg}$ & LC-MS/MS \\
\hline Amitraz * & Tespit Edilemedi & 0.010 & $\mathrm{mg} / \mathrm{kg}$ & LC-MS/MS \\
\hline Anilazine * & Tespit Edilemedi & 0.010 & $\mathrm{mg} / \mathrm{kg}$ & LC-MS/MS \\
\hline Anthraquinone & Tespit Edilemedi & 0.010 & $\mathrm{mg} / \mathrm{kg}$ & GC-MS \& GC-MS/MS \\
\hline Atrazine * & Tespit Edilemedi & 0.010 & $\mathrm{mg} / \mathrm{kg}$ & LC-MS/MS \\
\hline Azaconazole & Tespit Edilemedi & 0.010 & $\mathrm{mg} / \mathrm{kg}$ & LC-MS/MS \\
\hline Azadirachtin & Tespit Edilemedi & 0.010 & $\mathrm{mg} / \mathrm{kg}$ & LC-MS/MS \\
\hline Azinphos-ethyl * & Tespit Edilemedi & 0.010 & $\mathrm{mg} / \mathrm{kg}$ & GC-MS \& GC-MS/MS \\
\hline Azinphos-methyl & Tespit Edilemedi & 0.010 & $\mathrm{mg} / \mathrm{kg}$ & LC-MS/MS \\
\hline Azoxystrobin * & Tespit Edilemedi & 0.010 & $\mathrm{mg} / \mathrm{kg}$ & LC-MS/MS \\
\hline Barban * & Tespit Edilemedi & 0.010 & $\mathrm{mg} / \mathrm{kg}$ & LC-MS/MS \\
\hline Beflubutamid & Tespit Edilemedi & 0.010 & $\mathrm{mg} / \mathrm{kg}$ & GC-MS \& GC-MS/MS \\
\hline Benalaxyl ${ }^{*}$ & Tespit Edilemedi & 0.010 & $\mathrm{mg} / \mathrm{kg}$ & GC-MS \& GC-MS/MS \\
\hline Bendiocarb & Tespit Edilemedi & 0.010 & $\mathrm{mg} / \mathrm{kg}$ & LC-MS/MS \\
\hline Benfluralin (Benefin) & Tespit Edilemedi & 0.010 & $\mathrm{mg} / \mathrm{kg}$ & GC-MS \& GC-MS/MS \\
\hline Benfuracarb & Tespit Edilemedi & 0.010 & $\mathrm{mg} / \mathrm{kg}$ & LC-MS/MS \\
\hline Benodanil & Tespit Edilemedi & 0.010 & $\mathrm{mg} / \mathrm{kg}$ & LC-MS/MS \\
\hline Benomyl * & Tespit Edilemedi & 0.010 & $\mathrm{mg} / \mathrm{kg}$ & LC-MS/MS \\
\hline Benoxacor & Tespit Edilemedi & 0.010 & $\mathrm{mg} / \mathrm{kg}$ & GC-MS \& GC-MS/MS \\
\hline Bensulfuron-methyl & Tespit Edilemedi & 0.010 & $\mathrm{mg} / \mathrm{kg}$ & LC-MS/MS \\
\hline Bentazone ${ }^{*}$ & Tespit Edilemedi & 0.010 & $\mathrm{mg} / \mathrm{kg}$ & LC-MS/MS \\
\hline Benthiavalicarb-Isopropyl & Tespit Edilemedi & 0.010 & $\mathrm{mg} / \mathrm{kg}$ & LC-MS/MS \\
\hline Benzalkonium Chloride (BAC 10) * & Tespit Edilemedi & 0.010 & $\mathrm{mg} / \mathrm{kg}$ & LC-MS/MS \\
\hline Benzalkonium Chloride (BAC 12) * & Tespit Edilemedi & 0.010 & $\mathrm{mg} / \mathrm{kg}$ & LC-MS/MS \\
\hline Benzalkonium Chloride (BAC 14)* & Tespit Edilemedi & 0.010 & $\mathrm{mg} / \mathrm{kg}$ & LC-MS/MS \\
\hline Benzalkonium Chloride (BAC 16) * & Tespit Edilemedi & 0.010 & $\mathrm{mg} / \mathrm{kg}$ & LC-MS/MS \\
\hline beta-Cyfluthrin * & Tespit Edilemedi & 0.010 & $\mathrm{mg} / \mathrm{kg}$ & GC-MS \& GC-MS/MS \\
\hline Bifenazate & Tespit Edilemedi & 0.010 & $\mathrm{mg} / \mathrm{kg}$ & GC-MS \& GC-MS/MS \\
\hline Bifenox & Tespit Edilemedi & 0.010 & $\mathrm{mg} / \mathrm{kg}$ & GC-MS \& GC-MS/MS \\
\hline Bifenthrin * & Tespit Edilemedi & 0.010 & $\mathrm{mg} / \mathrm{kg}$ & GC-MS \& GC-MS/MS \\
\hline Binapacryl * & Tespit Edilemedi & 0.010 & $\mathrm{mg} / \mathrm{kg}$ & GC-MS \& GC-MS/MS \\
\hline Biphenyl (Diphenyl) & Tespit Edilemedi & 0.010 & $\mathrm{mg} / \mathrm{kg}$ & GC-MS \& GC-MS/MS \\
\hline Bitertanol * & Tespit Edilemedi & 0.010 & $\mathrm{mg} / \mathrm{kg}$ & GC-MS \& GC-MS/MS \\
\hline Boscalid (Nicobifen) ${ }^{*}$ & Tespit Edilemedi & 0.010 & $\mathrm{mg} / \mathrm{kg}$ & LC-MS/MS \\
\hline Bromacil & Tespit Edilemedi & 0.010 & $\mathrm{mg} / \mathrm{kg}$ & LC-MS/MS \\
\hline Bromfenvinphos & Tespit Edilemedi & 0.010 & $\mathrm{mg} / \mathrm{kg}$ & GC-MS \& GC-MS/MS \\
\hline Bromocyclen & Tespit Edilemedi & 0.010 & $\mathrm{mg} / \mathrm{kg}$ & GC-MS \& GC-MS/MS \\
\hline Bromophos-ethyl * & Tespit Edilemedi & 0.010 & $\mathrm{mg} / \mathrm{kg}$ & GC-MS \& GC-MS/MS \\
\hline Bromophos-methyl ${ }^{*}$ & Tespit Edilemedi & 0.010 & $\mathrm{mg} / \mathrm{kg}$ & GC-MS \& GC-MS/MS \\
\hline
\end{tabular}




\begin{tabular}{|c|c|c|c|c|}
\hline Bromopropylate * & Tespit Edilemedi & 0.010 & $\mathrm{mg} / \mathrm{kg}$ & GC-MS \& GC-MS/MS \\
\hline Bromoxynil & Tespit Edilemedi & 0.010 & $\mathrm{mg} / \mathrm{kg}$ & LC-MS/MS \\
\hline Bromuconazole * & Tespit Edilemedi & 0.010 & $\mathrm{mg} / \mathrm{kg}$ & LC-MS/MS \\
\hline Bupirimate * & Tespit Edilemedi & 0.010 & $\mathrm{mg} / \mathrm{kg}$ & LC-MS/MS \\
\hline Buprofezin * & Tespit Edilemedi & 0.010 & $\mathrm{mg} / \mathrm{kg}$ & LC-MS/MS \\
\hline Butafenacil & Tespit Edilemedi & 0.010 & $\mathrm{mg} / \mathrm{kg}$ & GC-MS \& GC-MS/MS \\
\hline Butocarboxim & Tespit Edilemedi & 0.010 & $\mathrm{mg} / \mathrm{kg}$ & LC-MS/MS \\
\hline Butocarboxim-Sulfoxide & Tespit Edilemedi & 0.010 & $\mathrm{mg} / \mathrm{kg}$ & LC-MS/MS \\
\hline Buturon & Tespit Edilemedi & 0.010 & $\mathrm{mg} / \mathrm{kg}$ & LC-MS/MS \\
\hline Butylate * & Tespit Edilemedi & 0.010 & $\mathrm{mg} / \mathrm{kg}$ & GC-MS \& GC-MS/MS \\
\hline Cadusafos & Tespit Edilemedi & 0.010 & $\mathrm{mg} / \mathrm{kg}$ & LC-MS/MS \\
\hline Captafol * & Tespit Edilemedi & 0.010 & $\mathrm{mg} / \mathrm{kg}$ & GC-MS \& GC-MS/MS \\
\hline Captan * & Tespit Edilemedi & 0.010 & $\mathrm{mg} / \mathrm{kg}$ & GC-MS \& GC-MS/MS \\
\hline Carbaryl * & Tespit Edilemedi & 0.010 & $\mathrm{mg} / \mathrm{kg}$ & LC-MS/MS \\
\hline Carbendazim * & Tespit Edilemedi & 0.010 & $\mathrm{mg} / \mathrm{kg}$ & LC-MS/MS \\
\hline Carbofuran * & Tespit Edilemedi & 0.010 & $\mathrm{mg} / \mathrm{kg}$ & LC-MS/MS \\
\hline Carbofuran-3-hydroxy & Tespit Edilemedi & 0.010 & $\mathrm{mg} / \mathrm{kg}$ & LC-MS/MS \\
\hline Carbophenothion & Tespit Edilemedi & 0.010 & $\mathrm{mg} / \mathrm{kg}$ & GC-MS \& GC-MS/MS \\
\hline Carbophenthion-methyl & Tespit Edilemedi & 0.010 & $\mathrm{mg} / \mathrm{kg}$ & GC-MS \& GC-MS/MS \\
\hline Carbosulfan * & Tespit Edilemedi & 0.010 & $\mathrm{mg} / \mathrm{kg}$ & GC-MS \& GC-MS/MS \\
\hline Carboxin * & Tespit Edilemedi & 0.010 & $\mathrm{mg} / \mathrm{kg}$ & LC-MS/MS \\
\hline Carfentrazone-ethyl & Tespit Edilemedi & 0.010 & $\mathrm{mg} / \mathrm{kg}$ & GC-MS \& GC-MS/MS \\
\hline \begin{tabular}{|l|} 
Chinomethionate \\
(Quinomethionate)
\end{tabular} & Tespit Edilemedi & 0.010 & $\mathrm{mg} / \mathrm{kg}$ & GC-MS \& GC-MS/MS \\
\hline Chlorantraniliprole & Tespit Edilemedi & 0.010 & $\mathrm{mg} / \mathrm{kg}$ & LC-MS/MS \\
\hline Chlorbenside * & Tespit Edilemedi & 0.010 & $\mathrm{mg} / \mathrm{kg}$ & GC-MS \& GC-MS/MS \\
\hline Chlorbromuron & Tespit Edilemedi & 0.010 & $\mathrm{mg} / \mathrm{kg}$ & LC-MS/MS \\
\hline Chlorbufam & Tespit Edilemedi & 0.010 & $\mathrm{mg} / \mathrm{kg}$ & LC-MS/MS \\
\hline Chlordane-cis (alpha) ${ }^{*}$ & Tespit Edilemedi & 0.010 & $\mathrm{mg} / \mathrm{kg}$ & GC-MS \& GC-MS/MS \\
\hline Chlordane-oxy & Tespit Edilemedi & 0.010 & $\mathrm{mg} / \mathrm{kg}$ & GC-MS \& GC-MS/MS \\
\hline Chlordane-trans (gamma) ${ }^{*}$ & Tespit Edilemedi & 0.010 & $\mathrm{mg} / \mathrm{kg}$ & GC-MS \& GC-MS/MS \\
\hline Chlorfenapyr & Tespit Edilemedi & 0.010 & $\mathrm{mg} / \mathrm{kg}$ & GC-MS \& GC-MS/MS \\
\hline Chlorfenson & Tespit Edilemedi & 0.010 & $\mathrm{mg} / \mathrm{kg}$ & GC-MS \& GC-MS/MS \\
\hline Chlorfenvinphos * & Tespit Edilemedi & 0.010 & $\mathrm{mg} / \mathrm{kg}$ & GC-MS \& GC-MS/MS \\
\hline Chlorfluazuron ${ }^{*}$ & Tespit Edilemedi & 0.010 & $\mathrm{mg} / \mathrm{kg}$ & LC-MS/MS \\
\hline Chloridazon * & Tespit Edilemedi & 0.010 & $\mathrm{mg} / \mathrm{kg}$ & LC-MS/MS \\
\hline \begin{tabular}{|l|} 
Chlormephos \\
\end{tabular} & Tespit Edilemedi & 0.010 & $\mathrm{mg} / \mathrm{kg}$ & GC-MS \& GC-MS/MS \\
\hline Chlormequat ${ }^{*}$ & Tespit Edilemedi & 0.010 & $\mathrm{mg} / \mathrm{kg}$ & LC-MS/MS \\
\hline Chlorobenzilate * & Tespit Edilemedi & 0.010 & $\mathrm{mg} / \mathrm{kg}$ & GC-MS \& GC-MS/MS \\
\hline Chloroneb & Tespit Edilemedi & 0.010 & $\mathrm{mg} / \mathrm{kg}$ & GC-MS \& GC-MS/MS \\
\hline Chloropropylate & Tespit Edilemedi & 0.010 & $\mathrm{mg} / \mathrm{kg}$ & GC-MS \& GC-MS/MS \\
\hline Chlorothalonil * & Tespit Edilemedi & 0.010 & $\mathrm{mg} / \mathrm{kg}$ & GC-MS \& GC-MS/MS \\
\hline Chlorotoluron * & Tespit Edilemedi & 0.010 & $\mathrm{mg} / \mathrm{kg}$ & LC-MS/MS \\
\hline \begin{tabular}{|l|} 
Chloroxuron \\
\end{tabular} & Tespit Edilemedi & 0.010 & $\mathrm{mg} / \mathrm{kg}$ & LC-MS/MS \\
\hline Chlorpropham * & Tespit Edilemedi & 0.010 & $\mathrm{mg} / \mathrm{kg}$ & LC-MS/MS \\
\hline Chlorpyrifos ${ }^{*}$ & Tespit Edilemedi & 0.010 & $\mathrm{mg} / \mathrm{kg}$ & GC-MS \& GC-MS/MS \\
\hline Chlorpyrifos-methyl * & Tespit Edilemedi & 0.010 & $\mathrm{mg} / \mathrm{kg}$ & GC-MS \& GC-MS/MS \\
\hline Chlorsulfuron & Tespit Edilemedi & 0.010 & $\mathrm{mg} / \mathrm{kg}$ & LC-MS/MS \\
\hline Chlorthal-dimethyl & Tespit Edilemedi & 0.010 & $\mathrm{mg} / \mathrm{kg}$ & GC-MS \& GC-MS/MS \\
\hline Chlorthion * & Tespit Edilemedi & 0.010 & $\mathrm{mg} / \mathrm{kg}$ & GC-MS \& GC-MS/MS \\
\hline Chlorthiophos & Tespit Edilemedi & 0.010 & $\mathrm{mg} / \mathrm{kg}$ & LC-MS/MS \\
\hline Chlozolinate & Tespit Edilemedi & 0.010 & $\mathrm{mg} / \mathrm{kg}$ & GC-MS \& GC-MS/MS \\
\hline Chromafenozide & Tespit Edilemedi & 0.010 & $\mathrm{mg} / \mathrm{kg}$ & LC-MS/MS \\
\hline Cinidon-ethyl * & Tespit Edilemedi & 0.010 & $\mathrm{mg} / \mathrm{kg}$ & LC-MS/MS \\
\hline Cinosulfuron & Tespit Edilemedi & 0.010 & $\mathrm{mg} / \mathrm{kg}$ & LC-MS/MS \\
\hline Clethodim * & Tespit Edilemedi & 0.010 & $\mathrm{mg} / \mathrm{kg}$ & LC-MS/MS \\
\hline Climbazole & Tespit Edilemedi & 0.010 & $\mathrm{mg} / \mathrm{kg}$ & LC-MS/MS \\
\hline Clodinafop-propargyl & Tespit Edilemedi & 0.010 & $\mathrm{mg} / \mathrm{kg}$ & LC-MS/MS \\
\hline Clofentezine * & Tespit Edilemedi & 0.010 & $\mathrm{mg} / \mathrm{kg}$ & LC-MS/MS \\
\hline Clomazone & Tespit Edilemedi & 0.010 & $\mathrm{mg} / \mathrm{kg}$ & LC-MS/MS \\
\hline Clopyralid & Tespit Edilemedi & 0.010 & $\mathrm{mg} / \mathrm{kg}$ & LC-MS/MS \\
\hline Cloquintocet-mexyl & Tespit Edilemedi & 0.010 & $\mathrm{mg} / \mathrm{kg}$ & LC-MS/MS \\
\hline Clothianidin & Tespit Edilemedi & 0.010 & $\mathrm{mg} / \mathrm{kg}$ & LC-MS/MS \\
\hline
\end{tabular}




\begin{tabular}{|c|c|c|c|c|}
\hline Coumaphos & Tespit Edilemedi & 0.010 & $\mathrm{mg} / \mathrm{kg}$ & GC-MS \& GC-MS/MS \\
\hline CPA, 4- & Tespit Edilemedi & 0.010 & $\mathrm{mg} / \mathrm{kg}$ & LC-MS/MS \\
\hline Crimidine & Tespit Edilemedi & 0.010 & $\mathrm{mg} / \mathrm{kg}$ & LC-MS/MS \\
\hline Cyanazine & Tespit Edilemedi & 0.010 & $\mathrm{mg} / \mathrm{kg}$ & LC-MS/MS \\
\hline Cyanofenphos & Tespit Edilemedi & 0.010 & $\mathrm{mg} / \mathrm{kg}$ & GC-MS \& GC-MS/MS \\
\hline Cyanophos * & Tespit Edilemedi & 0.010 & $\mathrm{mg} / \mathrm{kg}$ & GC-MS \& GC-MS/MS \\
\hline Cyazofamid & Tespit Edilemedi & 0.010 & $\mathrm{mg} / \mathrm{kg}$ & LC-MS/MS \\
\hline Cycloate ${ }^{*}$ & Tespit Edilemedi & 0.010 & $\mathrm{mg} / \mathrm{kg}$ & GC-MS \& GC-MS/MS \\
\hline Cycloxydim & Tespit Edilemedi & 0.010 & $\mathrm{mg} / \mathrm{kg}$ & LC-MS/MS \\
\hline Cyflufenamid & Tespit Edilemedi & 0.010 & $\mathrm{mg} / \mathrm{kg}$ & LC-MS/MS \\
\hline Cyfluthrin * & Tespit Edilemedi & 0.010 & $\mathrm{mg} / \mathrm{kg}$ & GC-MS \& GC-MS/MS \\
\hline Cyhalofop-butyl ${ }^{*}$ & Tespit Edilemedi & 0.010 & $\mathrm{mg} / \mathrm{kg}$ & GC-MS \& GC-MS/MS \\
\hline Cyhalothrin, lambda- ${ }^{*}$ & Tespit Edilemedi & 0.010 & $\mathrm{mg} / \mathrm{kg}$ & GC-MS \& GC-MS/MS \\
\hline Cyhexatin & Tespit Edilemedi & 0.010 & $\mathrm{mg} / \mathrm{kg}$ & LC-MS/MS \\
\hline Cymoxanil * & Tespit Edilemedi & 0.010 & $\mathrm{mg} / \mathrm{kg}$ & LC-MS/MS \\
\hline Cypermethrin ${ }^{*}$ & Tespit Edilemedi & 0.010 & $\mathrm{mg} / \mathrm{kg}$ & GC-MS \& GC-MS/MS \\
\hline Cyphenothrin & Tespit Edilemedi & 0.010 & $\mathrm{mg} / \mathrm{kg}$ & LC-MS/MS \\
\hline Cyproconazole * & Tespit Edilemedi & 0.010 & $\mathrm{mg} / \mathrm{kg}$ & LC-MS/MS \\
\hline Cyprodinil * & Tespit Edilemedi & 0.010 & $\mathrm{mg} / \mathrm{kg}$ & LC-MS/MS \\
\hline Cyromazine & Tespit Edilemedi & 0.010 & $\mathrm{mg} / \mathrm{kg}$ & LC-MS/MS \\
\hline $\mathrm{D}, 2,4^{-}$ & Tespit Edilemedi & 0.010 & $\mathrm{mg} / \mathrm{kg}$ & LC-MS/MS \\
\hline Daminozide $^{*}$ & Tespit Edilemedi & 0.010 & $\mathrm{mg} / \mathrm{kg}$ & LC-MS/MS \\
\hline Dazomet & Tespit Edilemedi & 0.010 & $\mathrm{mg} / \mathrm{kg}$ & LC-MS/MS \\
\hline $\begin{array}{l}\text { DDAC } \\
\text { (Didecyldimethylammonium } \\
\text { chloride) }{ }^{*}\end{array}$ & Tespit Edilemedi & 0.010 & $\mathrm{mg} / \mathrm{kg}$ & LC-MS/MS \\
\hline DDD, o-p * & Tespit Edilemedi & 0.010 & $\mathrm{mg} / \mathrm{kg}$ & GC-MS \& GC-MS/MS \\
\hline DDD, p-p * & Tespit Edilemedi & 0.010 & $\mathrm{mg} / \mathrm{kg}$ & GC-MS \& GC-MS/MS \\
\hline DDE, o-p * & Tespit Edilemedi & 0.010 & $\mathrm{mg} / \mathrm{kg}$ & GC-MS \& GC-MS/MS \\
\hline DDE, $p-p$ * & Tespit Edilemedi & 0.010 & $\mathrm{mg} / \mathrm{kg}$ & GC-MS \& GC-MS/MS \\
\hline DDT, o-p * & Tespit Edilemedi & 0.010 & $\mathrm{mg} / \mathrm{kg}$ & GC-MS \& GC-MS/MS \\
\hline DDT, p-p * & Tespit Edilemedi & 0.010 & $\mathrm{mg} / \mathrm{kg}$ & GC-MS \& GC-MS/MS \\
\hline DEET & Tespit Edilemedi & 0.010 & $\mathrm{mg} / \mathrm{kg}$ & GC-MS \& GC-MS/MS \\
\hline Deltamethrin * & Tespit Edilemedi & 0.010 & $\mathrm{mg} / \mathrm{kg}$ & LC-MS/MS \\
\hline Demeton-S-methyl ${ }^{*}$ & Tespit Edilemedi & 0.010 & $\mathrm{mg} / \mathrm{kg}$ & LC-MS/MS \\
\hline Demeton-S-methyl sulfone * & Tespit Edilemedi & 0.010 & $\mathrm{mg} / \mathrm{kg}$ & LC-MS/MS \\
\hline 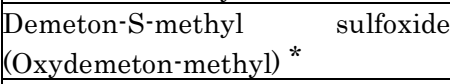 & Tespit Edilemedi & 0.010 & $\mathrm{mg} / \mathrm{kg}$ & LC-MS/MS \\
\hline Desmedipham * & Tespit Edilemedi & 0.010 & $\mathrm{mg} / \mathrm{kg}$ & LC-MS/MS \\
\hline Desmetryn & Tespit Edilemedi & 0.010 & $\mathrm{mg} / \mathrm{kg}$ & LC-MS/MS \\
\hline Diafenthiuron * & Tespit Edilemedi & 0.010 & $\mathrm{mg} / \mathrm{kg}$ & LC-MS/MS \\
\hline Diazinon * & Tespit Edilemedi & 0.010 & $\mathrm{mg} / \mathrm{kg}$ & GC-MS \& GC-MS/MS \\
\hline Dicamba & Tespit Edilemedi & 0.010 & $\mathrm{mg} / \mathrm{kg}$ & LC-MS/MS \\
\hline Dichlobenil & Tespit Edilemedi & 0.010 & $\mathrm{mg} / \mathrm{kg}$ & GC-MS \& GC-MS/MS \\
\hline Dichlofenthion & Tespit Edilemedi & 0.010 & $\mathrm{mg} / \mathrm{kg}$ & GC-MS \& GC-MS/MS \\
\hline Dichlofluanid * & Tespit Edilemedi & 0.010 & $\mathrm{mg} / \mathrm{kg}$ & LC-MS/MS \\
\hline Dichlorobenzophenone, 4,4- & Tespit Edilemedi & 0.010 & $\mathrm{mg} / \mathrm{kg}$ & GC-MS \& GC-MS/MS \\
\hline Dichlorvos (DDVP) ${ }^{*}$ & Tespit Edilemedi & 0.010 & $\mathrm{mg} / \mathrm{kg}$ & LC-MS/MS \\
\hline Diclobutrazol & Tespit Edilemedi & 0.010 & $\mathrm{mg} / \mathrm{kg}$ & GC-MS \& GC-MS/MS \\
\hline Diclofop-methyl * & Tespit Edilemedi & 0.010 & $\mathrm{mg} / \mathrm{kg}$ & GC-MS \& GC-MS/MS \\
\hline Dicloran & Tespit Edilemedi & 0.010 & $\mathrm{mg} / \mathrm{kg}$ & GC-MS \& GC-MS/MS \\
\hline Dicofol ${ }^{*}$ & Tespit Edilemedi & 0.010 & $\mathrm{mg} / \mathrm{kg}$ & GC-MS \& GC-MS/MS \\
\hline Dicrotophos & Tespit Edilemedi & 0.010 & $\mathrm{mg} / \mathrm{kg}$ & LC-MS/MS \\
\hline Dieldrin * & Tespit Edilemedi & 0.010 & $\mathrm{mg} / \mathrm{kg}$ & GC-MS \& GC-MS/MS \\
\hline Diethofencarb & Tespit Edilemedi & 0.010 & $\mathrm{mg} / \mathrm{kg}$ & LC-MS/MS \\
\hline Difenoconazole * & Tespit Edilemedi & 0.010 & $\mathrm{mg} / \mathrm{kg}$ & LC-MS/MS \\
\hline Diflubenzuron & Tespit Edilemedi & 0.010 & $\mathrm{mg} / \mathrm{kg}$ & LC-MS/MS \\
\hline Diflufenican & Tespit Edilemedi & 0.010 & $\mathrm{mg} / \mathrm{kg}$ & GC-MS \& GC-MS/MS \\
\hline Dimepiperate & Tespit Edilemedi & 0.010 & $\mathrm{mg} / \mathrm{kg}$ & LC-MS/MS \\
\hline Dimethachlor & Tespit Edilemedi & 0.010 & $\mathrm{mg} / \mathrm{kg}$ & GC-MS \& GC-MS/MS \\
\hline Dimethenamid * & Tespit Edilemedi & 0.010 & $\mathrm{mg} / \mathrm{kg}$ & LC-MS/MS \\
\hline Dimethipin * & Tespit Edilemedi & 0.010 & $\mathrm{mg} / \mathrm{kg}$ & GC-MS \& GC-MS/MS \\
\hline
\end{tabular}




\begin{tabular}{|c|c|c|c|c|}
\hline Dimethoate ${ }^{*}$ & Tespit Edilemedi & 0.010 & $\mathrm{mg} / \mathrm{kg}$ & LC-MS/MS \\
\hline Dimethomorph * & Tespit Edilemedi & 0.010 & $\mathrm{mg} / \mathrm{kg}$ & LC-MS/MS \\
\hline Dimetilan & Tespit Edilemedi & 0.010 & $\mathrm{mg} / \mathrm{kg}$ & LC-MS/MS \\
\hline Dimoxystrobin & Tespit Edilemedi & 0.010 & $\mathrm{mg} / \mathrm{kg}$ & LC-MS/MS \\
\hline Diniconazole & Tespit Edilemedi & 0.010 & $\mathrm{mg} / \mathrm{kg}$ & LC-MS/MS \\
\hline Dinobuton * & Tespit Edilemedi & 0.010 & $\mathrm{mg} / \mathrm{kg}$ & GC-MS \& GC-MS/MS \\
\hline Dinocap * & Tespit Edilemedi & 0.010 & $\mathrm{mg} / \mathrm{kg}$ & LC-MS/MS \\
\hline Dinotefuran & Tespit Edilemedi & 0.010 & $\mathrm{mg} / \mathrm{kg}$ & LC-MS/MS \\
\hline Dioxabenzofos (Salithion) ${ }^{*}$ & Tespit Edilemedi & 0.010 & $\mathrm{mg} / \mathrm{kg}$ & GC-MS \& GC-MS/MS \\
\hline Dioxacarb & Tespit Edilemedi & 0.010 & $\mathrm{mg} / \mathrm{kg}$ & LC-MS/MS \\
\hline Dioxathion & Tespit Edilemedi & 0.010 & $\mathrm{mg} / \mathrm{kg}$ & LC-MS/MS \\
\hline Diphenamid * & Tespit Edilemedi & 0.010 & $\mathrm{mg} / \mathrm{kg}$ & GC-MS \& GC-MS/MS \\
\hline Diphenylamine & Tespit Edilemedi & 0.010 & $\mathrm{mg} / \mathrm{kg}$ & GC-MS \& GC-MS/MS \\
\hline Dipropetryn & Tespit Edilemedi & 0.010 & $\mathrm{mg} / \mathrm{kg}$ & LC-MS/MS \\
\hline Disulfoton & Tespit Edilemedi & 0.010 & $\mathrm{mg} / \mathrm{kg}$ & GC-MS \& GC-MS/MS \\
\hline Disulfoton-Sulfone & Tespit Edilemedi & 0.010 & $\mathrm{mg} / \mathrm{kg}$ & LC-MS/MS \\
\hline Disulfoton-Sulfoxide & Tespit Edilemedi & 0.010 & $\mathrm{mg} / \mathrm{kg}$ & LC-MS/MS \\
\hline Ditalimfos & Tespit Edilemedi & 0.010 & $\mathrm{mg} / \mathrm{kg}$ & LC-MS/MS \\
\hline Dithianon * & Tespit Edilemedi & 0.010 & $\mathrm{mg} / \mathrm{kg}$ & LC-MS/MS \\
\hline Diuron * & Tespit Edilemedi & 0.010 & $\mathrm{mg} / \mathrm{kg}$ & LC-MS/MS \\
\hline DMF (Amitraz Metab.) & Tespit Edilemedi & 0.010 & $\mathrm{mg} / \mathrm{kg}$ & LC-MS/MS \\
\hline DMPF (Amitraz Metab.) & Tespit Edilemedi & 0.010 & $\mathrm{mg} / \mathrm{kg}$ & LC-MS/MS \\
\hline Dodemorph * & Tespit Edilemedi & 0.010 & $\mathrm{mg} / \mathrm{kg}$ & GC-MS \& GC-MS/MS \\
\hline Dodine ${ }^{*}$ & Tespit Edilemedi & 0.010 & $\mathrm{mg} / \mathrm{kg}$ & LC-MS/MS \\
\hline Emamectin B1a & Tespit Edilemedi & 0.010 & $\mathrm{mg} / \mathrm{kg}$ & LC-MS/MS \\
\hline Emamectin B1b & Tespit Edilemedi & 0.010 & $\mathrm{mg} / \mathrm{kg}$ & LC-MS/MS \\
\hline Emamectin Total & Tespit Edilemedi & 0.010 & $\mathrm{mg} / \mathrm{kg}$ & LC-MS/MS \\
\hline Endosulfan, alpha- ${ }^{*}$ & Tespit Edilemedi & 0.010 & $\mathrm{mg} / \mathrm{kg}$ & GC-MS \& GC-MS/MS \\
\hline Endosulfan, beta ${ }^{*}$ & Tespit Edilemedi & 0.010 & $\mathrm{mg} / \mathrm{kg}$ & GC-MS \& GC-MS/MS \\
\hline Endosulfan-sulfate ${ }^{*}$ & Tespit Edilemedi & 0.010 & $\mathrm{mg} / \mathrm{kg}$ & GC-MS \& GC-MS/MS \\
\hline Endrin * & Tespit Edilemedi & 0.010 & $\mathrm{mg} / \mathrm{kg}$ & GC-MS \& GC-MS/MS \\
\hline Endrin aldehyde * & Tespit Edilemedi & 0.010 & $\mathrm{mg} / \mathrm{kg}$ & GC-MS \& GC-MS/MS \\
\hline $\mathrm{EPN}^{*}$ & Tespit Edilemedi & 0.010 & $\mathrm{mg} / \mathrm{kg}$ & GC-MS \& GC-MS/MS \\
\hline Epoxiconazole * & Tespit Edilemedi & 0.010 & $\mathrm{mg} / \mathrm{kg}$ & LC-MS/MS \\
\hline EPTC & Tespit Edilemedi & 0.010 & $\mathrm{mg} / \mathrm{kg}$ & GC-MS \& GC-MS/MS \\
\hline Esfenvalerate ${ }^{*}$ & Tespit Edilemedi & 0.010 & $\mathrm{mg} / \mathrm{kg}$ & GC-MS \& GC-MS/MS \\
\hline Etaconazole & Tespit Edilemedi & 0.010 & $\mathrm{mg} / \mathrm{kg}$ & GC-MS \& GC-MS/MS \\
\hline Ethalfluralin * & Tespit Edilemedi & 0.010 & $\mathrm{mg} / \mathrm{kg}$ & GC-MS \& GC-MS/MS \\
\hline Ethiofencarb * & Tespit Edilemedi & 0.010 & $\mathrm{mg} / \mathrm{kg}$ & LC-MS/MS \\
\hline Ethiofencarb-Sulfone & Tespit Edilemedi & 0.010 & $\mathrm{mg} / \mathrm{kg}$ & LC-MS/MS \\
\hline Ethiofencarb-Sulfoxide & Tespit Edilemedi & 0.010 & $\mathrm{mg} / \mathrm{kg}$ & LC-MS/MS \\
\hline Ethion & Tespit Edilemedi & 0.010 & $\mathrm{mg} / \mathrm{kg}$ & GC-MS \& GC-MS/MS \\
\hline Ethirimol & Tespit Edilemedi & 0.010 & $\mathrm{mg} / \mathrm{kg}$ & LC-MS/MS \\
\hline Ethofumesate ${ }^{*}$ & Tespit Edilemedi & 0.010 & $\mathrm{mg} / \mathrm{kg}$ & LC-MS/MS \\
\hline Ethoprophos * & Tespit Edilemedi & 0.010 & $\mathrm{mg} / \mathrm{kg}$ & GC-MS \& GC-MS/MS \\
\hline Ethoxyquin & Tespit Edilemedi & 0.010 & $\mathrm{mg} / \mathrm{kg}$ & GC-MS \& GC-MS/MS \\
\hline Etofenprox * & Tespit Edilemedi & 0.010 & $\mathrm{mg} / \mathrm{kg}$ & LC-MS/MS \\
\hline Etoxazole & Tespit Edilemedi & 0.010 & $\mathrm{mg} / \mathrm{kg}$ & LC-MS/MS \\
\hline Etridiazole & Tespit Edilemedi & 0.010 & $\mathrm{mg} / \mathrm{kg}$ & GC-MS \& GC-MS/MS \\
\hline Etrimfos * & Tespit Edilemedi & 0.010 & $\mathrm{mg} / \mathrm{kg}$ & GC-MS \& GC-MS/MS \\
\hline Famoxadone * & Tespit Edilemedi & 0.010 & $\mathrm{mg} / \mathrm{kg}$ & LC-MS/MS \\
\hline Famphur (Famophos) & Tespit Edilemedi & 0.010 & $\mathrm{mg} / \mathrm{kg}$ & LC-MS/MS \\
\hline Fenamidone ${ }^{*}$ & Tespit Edilemedi & 0.010 & $\mathrm{mg} / \mathrm{kg}$ & LC-MS/MS \\
\hline Fenamiphos * & Tespit Edilemedi & 0.010 & $\mathrm{mg} / \mathrm{kg}$ & GC-MS \& GC-MS/MS \\
\hline Fenarimol * & Tespit Edilemedi & 0.010 & $\mathrm{mg} / \mathrm{kg}$ & LC-MS/MS \\
\hline Fenazaquin * & Tespit Edilemedi & 0.010 & $\mathrm{mg} / \mathrm{kg}$ & LC-MS/MS \\
\hline Fenbuconazole & Tespit Edilemedi & 0.010 & $\mathrm{mg} / \mathrm{kg}$ & LC-MS/MS \\
\hline Fenbutatin-oxide & Tespit Edilemedi & 0.010 & $\mathrm{mg} / \mathrm{kg}$ & LC-MS/MS \\
\hline Fenchlorphos * & Tespit Edilemedi & 0.010 & $\mathrm{mg} / \mathrm{kg}$ & GC-MS \& GC-MS/MS \\
\hline Fenfluthrin & Tespit Edilemedi & 0.010 & $\mathrm{mg} / \mathrm{kg}$ & GC-MS \& GC-MS/MS \\
\hline Fenhexamid * & Tespit Edilemedi & 0.010 & $\mathrm{mg} / \mathrm{kg}$ & LC-MS/MS \\
\hline Fenitrothion * & Tespit Edilemedi & 0.010 & $\mathrm{mg} / \mathrm{kg}$ & GC-MS \& GC-MS/MS \\
\hline
\end{tabular}




\begin{tabular}{|c|c|c|c|c|}
\hline Fenobucarb & Tespit Edilemedi & 0.010 & $\mathrm{mg} / \mathrm{kg}$ & LC-MS/MS \\
\hline Fenoxaprop-P-ethyl & Tespit Edilemedi & 0.010 & $\mathrm{mg} / \mathrm{kg}$ & LC-MS/MS \\
\hline Fenoxycarb * & Tespit Edilemedi & 0.010 & $\mathrm{mg} / \mathrm{kg}$ & LC-MS/MS \\
\hline Fenpiclonil & Tespit Edilemedi & 0.010 & $\mathrm{mg} / \mathrm{kg}$ & LC-MS/MS \\
\hline Fenpropathrin * & Tespit Edilemedi & 0.010 & $\mathrm{mg} / \mathrm{kg}$ & LC-MS/MS \\
\hline Fenpropidin & Tespit Edilemedi & 0.010 & $\mathrm{mg} / \mathrm{kg}$ & GC-MS \& GC-MS/MS \\
\hline Fenpropimorph & Tespit Edilemedi & 0.010 & $\mathrm{mg} / \mathrm{kg}$ & LC-MS/MS \\
\hline Fenpyroximate ${ }^{*}$ & Tespit Edilemedi & 0.010 & $\mathrm{mg} / \mathrm{kg}$ & LC-MS/MS \\
\hline Fenson (CPBS,PCPBS) & Tespit Edilemedi & 0.010 & $\mathrm{mg} / \mathrm{kg}$ & GC-MS \& GC-MS/MS \\
\hline Fensulfothion & Tespit Edilemedi & 0.010 & $\mathrm{mg} / \mathrm{kg}$ & LC-MS/MS \\
\hline Fenthion * & Tespit Edilemedi & 0.010 & $\mathrm{mg} / \mathrm{kg}$ & LC-MS/MS \\
\hline Fenthion-oxon & Tespit Edilemedi & 0.010 & $\mathrm{mg} / \mathrm{kg}$ & LC-MS/MS \\
\hline Fenthion-oxon Sulfone * & Tespit Edilemedi & 0.010 & $\mathrm{mg} / \mathrm{kg}$ & LC-MS/MS \\
\hline Fenthion-oxon Sulfoxide & Tespit Edilemedi & 0.010 & $\mathrm{mg} / \mathrm{kg}$ & LC-MS/MS \\
\hline Fenthion-Sulfone & Tespit Edilemedi & 0.010 & $\mathrm{mg} / \mathrm{kg}$ & LC-MS/MS \\
\hline Fenthion-Sulfoxide & Tespit Edilemedi & 0.010 & $\mathrm{mg} / \mathrm{kg}$ & LC-MS/MS \\
\hline Fentin Acetate & Tespit Edilemedi & 0.010 & $\mathrm{mg} / \mathrm{kg}$ & LC-MS/MS \\
\hline Fenuron & Tespit Edilemedi & 0.010 & $\mathrm{mg} / \mathrm{kg}$ & LC-MS/MS \\
\hline Fenvalerate ${ }^{*}$ & Tespit Edilemedi & 0.010 & $\mathrm{mg} / \mathrm{kg}$ & GC-MS \& GC-MS/MS \\
\hline Fipronil * & Tespit Edilemedi & 0.010 & $\mathrm{mg} / \mathrm{kg}$ & GC-MS \& GC-MS/MS \\
\hline Fipronil-Desulfinyl & Tespit Edilemedi & 0.010 & $\mathrm{mg} / \mathrm{kg}$ & LC-MS/MS \\
\hline Fipronil-Sulfide & Tespit Edilemedi & 0.010 & $\mathrm{mg} / \mathrm{kg}$ & LC-MS/MS \\
\hline Fipronil-Sulfone & Tespit Edilemedi & 0.010 & $\mathrm{mg} / \mathrm{kg}$ & LC-MS/MS \\
\hline Flamprop-Isopropyl & Tespit Edilemedi & 0.010 & $\mathrm{mg} / \mathrm{kg}$ & LC-MS/MS \\
\hline Flamprop-methyl & Tespit Edilemedi & 0.010 & $\mathrm{mg} / \mathrm{kg}$ & LC-MS/MS \\
\hline Flonicamid & Tespit Edilemedi & 0.010 & $\mathrm{mg} / \mathrm{kg}$ & LC-MS/MS \\
\hline Florasulam & Tespit Edilemedi & 0.010 & $\mathrm{mg} / \mathrm{kg}$ & LC-MS/MS \\
\hline Fluazifop (free acid) & Tespit Edilemedi & 0.010 & $\mathrm{mg} / \mathrm{kg}$ & LC-MS/MS \\
\hline Fluazifop-butyl & Tespit Edilemedi & 0.010 & $\mathrm{mg} / \mathrm{kg}$ & LC-MS/MS \\
\hline Fluazifop-P (free acid) & Tespit Edilemedi & 0.010 & $\mathrm{mg} / \mathrm{kg}$ & LC-MS/MS \\
\hline Fluazifop-P-butyl * & Tespit Edilemedi & 0.010 & $\mathrm{mg} / \mathrm{kg}$ & GC-MS \& GC-MS/MS \\
\hline Fluazinam & Tespit Edilemedi & 0.010 & $\mathrm{mg} / \mathrm{kg}$ & LC-MS/MS \\
\hline Fluazuron & Tespit Edilemedi & 0.010 & $\mathrm{mg} / \mathrm{kg}$ & LC-MS/MS \\
\hline Flubendiamide & Tespit Edilemedi & 0.010 & $\mathrm{mg} / \mathrm{kg}$ & LC-MS/MS \\
\hline Flubenzimine & Tespit Edilemedi & 0.010 & $\mathrm{mg} / \mathrm{kg}$ & LC-MS/MS \\
\hline Fluchloralin & Tespit Edilemedi & 0.010 & $\mathrm{mg} / \mathrm{kg}$ & GC-MS \& GC-MS/MS \\
\hline Flucythrinate ${ }^{*}$ & Tespit Edilemedi & 0.010 & $\mathrm{mg} / \mathrm{kg}$ & GC-MS \& GC-MS/MS \\
\hline Fludioxonil ${ }^{*}$ & Tespit Edilemedi & 0.010 & $\mathrm{mg} / \mathrm{kg}$ & LC-MS/MS \\
\hline Flufenacet & Tespit Edilemedi & 0.010 & $\mathrm{mg} / \mathrm{kg}$ & LC-MS/MS \\
\hline Flufenoxuron & Tespit Edilemedi & 0.010 & $\mathrm{mg} / \mathrm{kg}$ & LC-MS/MS \\
\hline Flumioxazin & Tespit Edilemedi & 0.010 & $\mathrm{mg} / \mathrm{kg}$ & GC-MS \& GC-MS/MS \\
\hline Fluometuron & Tespit Edilemedi & 0.010 & $\mathrm{mg} / \mathrm{kg}$ & LC-MS/MS \\
\hline Fluopicolide & Tespit Edilemedi & 0.010 & $\mathrm{mg} / \mathrm{kg}$ & LC-MS/MS \\
\hline Fluopyram * & Tespit Edilemedi & 0.010 & $\mathrm{mg} / \mathrm{kg}$ & GC-MS \& GC-MS/MS \\
\hline Fluotrimazole & Tespit Edilemedi & 0.010 & $\mathrm{mg} / \mathrm{kg}$ & GC-MS \& GC-MS/MS \\
\hline Fluoxastrobin & Tespit Edilemedi & 0.010 & $\mathrm{mg} / \mathrm{kg}$ & LC-MS/MS \\
\hline Fluquinconazole & Tespit Edilemedi & 0.010 & $\mathrm{mg} / \mathrm{kg}$ & LC-MS/MS \\
\hline Flurochloridone * & Tespit Edilemedi & 0.010 & $\mathrm{mg} / \mathrm{kg}$ & GC-MS \& GC-MS/MS \\
\hline Fluroxypyr-meptyl & Tespit Edilemedi & 0.010 & $\mathrm{mg} / \mathrm{kg}$ & LC-MS/MS \\
\hline Flurprimidol & Tespit Edilemedi & 0.010 & $\mathrm{mg} / \mathrm{kg}$ & GC-MS \& GC-MS/MS \\
\hline Flusilazole & Tespit Edilemedi & 0.010 & $\mathrm{mg} / \mathrm{kg}$ & LC-MS/MS \\
\hline Fluthiacet-methyl & Tespit Edilemedi & 0.010 & $\mathrm{mg} / \mathrm{kg}$ & LC-MS/MS \\
\hline Flutolanil & Tespit Edilemedi & 0.010 & $\mathrm{mg} / \mathrm{kg}$ & GC-MS \& GC-MS/MS \\
\hline Flutriafol ${ }^{*}$ & Tespit Edilemedi & 0.010 & $\mathrm{mg} / \mathrm{kg}$ & LC-MS/MS \\
\hline Folpet ${ }^{*}$ & Tespit Edilemedi & 0.010 & $\mathrm{mg} / \mathrm{kg}$ & GC-MS \& GC-MS/MS \\
\hline Fonofos & Tespit Edilemedi & 0.010 & $\mathrm{mg} / \mathrm{kg}$ & LC-MS/MS \\
\hline Foramsulfuron & Tespit Edilemedi & 0.010 & $\mathrm{mg} / \mathrm{kg}$ & LC-MS/MS \\
\hline Forchlorfenuron & Tespit Edilemedi & 0.010 & $\mathrm{mg} / \mathrm{kg}$ & LC-MS/MS \\
\hline Formetanate (hydrochloride) & Tespit Edilemedi & 0.010 & $\mathrm{mg} / \mathrm{kg}$ & LC-MS/MS \\
\hline Formothion * & Tespit Edilemedi & 0.010 & $\mathrm{mg} / \mathrm{kg}$ & GC-MS \& GC-MS/MS \\
\hline Fosthiazate & Tespit Edilemedi & 0.010 & $\mathrm{mg} / \mathrm{kg}$ & LC-MS/MS \\
\hline Fuberidazole & Tespit Edilemedi & 0.010 & $\mathrm{mg} / \mathrm{kg}$ & LC-MS/MS \\
\hline Furalaxyl & Tespit Edilemedi & 0.010 & $\mathrm{mg} / \mathrm{kg}$ & LC-MS/MS \\
\hline Furathiocarb * & Tespit Edilemedi & 0.010 & $\mathrm{mg} / \mathrm{kg}$ & LC-MS/MS \\
\hline Halfenprox & Tespit Edilemedi & 0.010 & $\mathrm{mg} / \mathrm{kg}$ & GC-MS \& GC-MS/MS \\
\hline
\end{tabular}




\begin{tabular}{|c|c|c|c|c|}
\hline Halosulfuron-methyl ${ }^{*}$ & Tespit Edilemedi & 0.010 & $\mathrm{mg} / \mathrm{kg}$ & LC-MS/MS \\
\hline Haloxyfop & Tespit Edilemedi & 0.010 & $\mathrm{mg} / \mathrm{kg}$ & LC-MS/MS \\
\hline Haloxyfop-2-ethoxyethyl & Tespit Edilemedi & 0.010 & $\mathrm{mg} / \mathrm{kg}$ & LC-MS/MS \\
\hline Haloxyfop-methyl & Tespit Edilemedi & 0.010 & $\mathrm{mg} / \mathrm{kg}$ & LC-MS/MS \\
\hline Haloxyfop-r-methyl ester * & Tespit Edilemedi & 0.010 & $\mathrm{mg} / \mathrm{kg}$ & LC-MS/MS \\
\hline $\mathrm{HCH}$, alpha $^{*}{ }^{*}$ & Tespit Edilemedi & 0.010 & $\mathrm{mg} / \mathrm{kg}$ & GC-MS \& GC-MS/MS \\
\hline $\mathrm{HCH}$, beta $^{*}$ & Tespit Edilemedi & 0.010 & $\mathrm{mg} / \mathrm{kg}$ & GC-MS \& GC-MS/MS \\
\hline $\mathrm{HCH}$, delta $^{*}{ }^{*}$ & Tespit Edilemedi & 0.010 & $\mathrm{mg} / \mathrm{kg}$ & GC-MS \& GC-MS/MS \\
\hline $\mathrm{HCH}$, epsilon- & Tespit Edilemedi & 0.010 & $\mathrm{mg} / \mathrm{kg}$ & GC-MS \& GC-MS/MS \\
\hline Heptachlor * & Tespit Edilemedi & 0.010 & $\mathrm{mg} / \mathrm{kg}$ & GC-MS \& GC-MS/MS \\
\hline $\begin{array}{l}\text { Heptachlor endo-epoxide (trans } \\
\text { isomer) }{ }^{*}\end{array}$ & Tespit Edilemedi & 0.010 & $\mathrm{mg} / \mathrm{kg}$ & GC-MS \& GC-MS/MS \\
\hline Heptachlor exo-epoxide (cis isomer & Tespit Edilemedi & 0.010 & $\mathrm{mg} / \mathrm{kg}$ & GC-MS \& GC-MS/MS \\
\hline Heptenophos & Tespit Edilemedi & 0.010 & $\mathrm{mg} / \mathrm{kg}$ & LC-MS/MS \\
\hline Hexachlorobenzene ${ }^{*}$ & Tespit Edilemedi & 0.010 & $\mathrm{mg} / \mathrm{kg}$ & GC-MS \& GC-MS/MS \\
\hline Hexaconazole ${ }^{*}$ & Tespit Edilemedi & 0.010 & $\mathrm{mg} / \mathrm{kg}$ & LC-MS/MS \\
\hline Hexaflumuron & Tespit Edilemedi & 0.010 & $\mathrm{mg} / \mathrm{kg}$ & LC-MS/MS \\
\hline Hexazinone & Tespit Edilemedi & 0.010 & $\mathrm{mg} / \mathrm{kg}$ & LC-MS/MS \\
\hline Hexythiazox ${ }^{*}$ & Tespit Edilemedi & 0.010 & $\mathrm{mg} / \mathrm{kg}$ & LC-MS/MS \\
\hline Imazalil * & Tespit Edilemedi & 0.010 & $\mathrm{mg} / \mathrm{kg}$ & LC-MS/MS \\
\hline Imazamox * & Tespit Edilemedi & 0.010 & $\mathrm{mg} / \mathrm{kg}$ & LC-MS/MS \\
\hline Imazapyr ${ }^{*}$ & Tespit Edilemedi & 0.010 & $\mathrm{mg} / \mathrm{kg}$ & LC-MS/MS \\
\hline Imazaquin & Tespit Edilemedi & 0.010 & $\mathrm{mg} / \mathrm{kg}$ & LC-MS/MS \\
\hline Imazethapyr & Tespit Edilemedi & 0.010 & $\mathrm{mg} / \mathrm{kg}$ & LC-MS/MS \\
\hline Imazosulfuron & Tespit Edilemedi & 0.010 & $\mathrm{mg} / \mathrm{kg}$ & LC-MS/MS \\
\hline Imibenconazole & Tespit Edilemedi & 0.010 & $\mathrm{mg} / \mathrm{kg}$ & LC-MS/MS \\
\hline Imidacloprid * & Tespit Edilemedi & 0.010 & $\mathrm{mg} / \mathrm{kg}$ & LC-MS/MS \\
\hline Indoxacarb & Tespit Edilemedi & 0.010 & $\mathrm{mg} / \mathrm{kg}$ & LC-MS/MS \\
\hline Iodofenphos (Jodfenphos) & Tespit Edilemedi & 0.010 & $\mathrm{mg} / \mathrm{kg}$ & GC-MS \& GC-MS/MS \\
\hline Iodosulfuron-methyl & Tespit Edilemedi & 0.010 & $\mathrm{mg} / \mathrm{kg}$ & LC-MS/MS \\
\hline Ioxynil * & Tespit Edilemedi & 0.010 & $\mathrm{mg} / \mathrm{kg}$ & LC-MS/MS \\
\hline Iprobenfos & Tespit Edilemedi & 0.010 & $\mathrm{mg} / \mathrm{kg}$ & GC-MS \& GC-MS/MS \\
\hline Iprodione * & Tespit Edilemedi & 0.010 & $\mathrm{mg} / \mathrm{kg}$ & GC-MS \& GC-MS/MS \\
\hline Iprovalicarb & Tespit Edilemedi & 0.010 & $\mathrm{mg} / \mathrm{kg}$ & LC-MS/MS \\
\hline Isazofos * & Tespit Edilemedi & 0.010 & $\mathrm{mg} / \mathrm{kg}$ & GC-MS \& GC-MS/MS \\
\hline Isocarbamid & Tespit Edilemedi & 0.010 & $\mathrm{mg} / \mathrm{kg}$ & LC-MS/MS \\
\hline Isocarbophos & Tespit Edilemedi & 0.010 & $\mathrm{mg} / \mathrm{kg}$ & GC-MS \& GC-MS/MS \\
\hline Isodrin & Tespit Edilemedi & 0.010 & $\mathrm{mg} / \mathrm{kg}$ & GC-MS \& GC-MS/MS \\
\hline Isofenphos & Tespit Edilemedi & 0.010 & $\mathrm{mg} / \mathrm{kg}$ & GC-MS \& GC-MS/MS \\
\hline Isofenphos-methyl & Tespit Edilemedi & 0.010 & $\mathrm{mg} / \mathrm{kg}$ & LC-MS/MS \\
\hline Isofenphos-oxon & Tespit Edilemedi & 0.010 & $\mathrm{mg} / \mathrm{kg}$ & LC-MS/MS \\
\hline Isoprocarb & Tespit Edilemedi & 0.010 & $\mathrm{mg} / \mathrm{kg}$ & LC-MS/MS \\
\hline Isoprothiolane & Tespit Edilemedi & 0.010 & $\mathrm{mg} / \mathrm{kg}$ & LC-MS/MS \\
\hline Isoproturon & Tespit Edilemedi & 0.010 & $\mathrm{mg} / \mathrm{kg}$ & LC-MS/MS \\
\hline Isoxaben & Tespit Edilemedi & 0.010 & $\mathrm{mg} / \mathrm{kg}$ & LC-MS/MS \\
\hline Isoxadifen-ethyl & Tespit Edilemedi & 0.010 & $\mathrm{mg} / \mathrm{kg}$ & LC-MS/MS \\
\hline Isoxaflutole & Tespit Edilemedi & 0.010 & $\mathrm{mg} / \mathrm{kg}$ & LC-MS/MS \\
\hline Isoxathion & Tespit Edilemedi & 0.010 & $\mathrm{mg} / \mathrm{kg}$ & LC-MS/MS \\
\hline Kresoxim-methyl * & Tespit Edilemedi & 0.010 & $\mathrm{mg} / \mathrm{kg}$ & LC-MS/MS \\
\hline Lenacil * & Tespit Edilemedi & 0.010 & $\mathrm{mg} / \mathrm{kg}$ & LC-MS/MS \\
\hline Leptophos & Tespit Edilemedi & 0.010 & $\mathrm{mg} / \mathrm{kg}$ & GC-MS \& GC-MS/MS \\
\hline Lindane $(\mathrm{HCH}, \text { gamma })^{*}{ }^{*}$ & Tespit Edilemedi & 0.010 & $\mathrm{mg} / \mathrm{kg}$ & GC-MS \& GC-MS/MS \\
\hline Linuron & Tespit Edilemedi & 0.010 & $\mathrm{mg} / \mathrm{kg}$ & LC-MS/MS \\
\hline Lufenuron * & Tespit Edilemedi & 0.010 & $\mathrm{mg} / \mathrm{kg}$ & LC-MS/MS \\
\hline Malaoxon * & Tespit Edilemedi & 0.010 & $\mathrm{mg} / \mathrm{kg}$ & LC-MS/MS \\
\hline Malathion * & Tespit Edilemedi & 0.010 & $\mathrm{mg} / \mathrm{kg}$ & LC-MS/MS \\
\hline Mandipropamid & Tespit Edilemedi & 0.010 & $\mathrm{mg} / \mathrm{kg}$ & LC-MS/MS \\
\hline MCPA & Tespit Edilemedi & 0.010 & $\mathrm{mg} / \mathrm{kg}$ & LC-MS/MS \\
\hline Mecarbam & Tespit Edilemedi & 0.010 & $\mathrm{mg} / \mathrm{kg}$ & LC-MS/MS \\
\hline Mefenpyr-Diethyl * & Tespit Edilemedi & 0.010 & $\mathrm{mg} / \mathrm{kg}$ & GC-MS \& GC-MS/MS \\
\hline Mepanipyrim & Tespit Edilemedi & 0.010 & $\mathrm{mg} / \mathrm{kg}$ & LC-MS/MS \\
\hline Mepiquat & Tespit Edilemedi & 0.010 & $\mathrm{mg} / \mathrm{kg}$ & LC-MS/MS \\
\hline Mepronil & Tespit Edilemedi & 0.010 & $\mathrm{mg} / \mathrm{kg}$ & GC-MS \& GC-MS/MS \\
\hline Mesosulfuron-methyl & Tespit Edilemedi & 0.010 & $\mathrm{mg} / \mathrm{kg}$ & LC-MS/MS \\
\hline
\end{tabular}




\begin{tabular}{|c|c|c|c|c|}
\hline Mesotrione & Tespit Edilemedi & 0.010 & $\mathrm{mg} / \mathrm{kg}$ & LC-MS/MS \\
\hline Metaflumizone & Tespit Edilemedi & 0.010 & $\mathrm{mg} / \mathrm{kg}$ & LC-MS/MS \\
\hline Metalaxyl & Tespit Edilemedi & 0.010 & $\mathrm{mg} / \mathrm{kg}$ & GC-MS \& GC-MS/MS \\
\hline Metalaxyl-M * & Tespit Edilemedi & 0.010 & $\mathrm{mg} / \mathrm{kg}$ & LC-MS/MS \\
\hline Metamitron * & Tespit Edilemedi & 0.010 & $\mathrm{mg} / \mathrm{kg}$ & LC-MS/MS \\
\hline Metazachlor * & Tespit Edilemedi & 0.010 & $\mathrm{mg} / \mathrm{kg}$ & GC-MS \& GC-MS/MS \\
\hline Metconazole & Tespit Edilemedi & 0.010 & $\mathrm{mg} / \mathrm{kg}$ & LC-MS/MS \\
\hline Methabenzthiazuron & Tespit Edilemedi & 0.010 & $\mathrm{mg} / \mathrm{kg}$ & LC-MS/MS \\
\hline Methacrifos & Tespit Edilemedi & 0.010 & $\mathrm{mg} / \mathrm{kg}$ & LC-MS/MS \\
\hline Methamidophos ${ }^{*}$ & Tespit Edilemedi & 0.010 & $\mathrm{mg} / \mathrm{kg}$ & LC-MS/MS \\
\hline Methidathion ${ }^{*}$ & Tespit Edilemedi & 0.010 & $\mathrm{mg} / \mathrm{kg}$ & GC-MS \& GC-MS/MS \\
\hline Methiocarb * & Tespit Edilemedi & 0.010 & $\mathrm{mg} / \mathrm{kg}$ & LC-MS/MS \\
\hline Methiocarb-sulfone & Tespit Edilemedi & 0.010 & $\mathrm{mg} / \mathrm{kg}$ & LC-MS/MS \\
\hline Methiocarb-sulfoxide & Tespit Edilemedi & 0.010 & $\mathrm{mg} / \mathrm{kg}$ & LC-MS/MS \\
\hline Methomyl & Tespit Edilemedi & 0.010 & $\mathrm{mg} / \mathrm{kg}$ & LC-MS/MS \\
\hline Methoprotryne & Tespit Edilemedi & 0.010 & $\mathrm{mg} / \mathrm{kg}$ & LC-MS/MS \\
\hline Methoxychlor ${ }^{*}$ & Tespit Edilemedi & 0.010 & $\mathrm{mg} / \mathrm{kg}$ & GC-MS \& GC-MS/MS \\
\hline Methoxyfenozide & Tespit Edilemedi & 0.010 & $\mathrm{mg} / \mathrm{kg}$ & LC-MS/MS \\
\hline Metobromuron & Tespit Edilemedi & 0.010 & $\mathrm{mg} / \mathrm{kg}$ & LC-MS/MS \\
\hline Metolachlor * & Tespit Edilemedi & 0.010 & $\mathrm{mg} / \mathrm{kg}$ & GC-MS \& GC-MS/MS \\
\hline Metolcarb & Tespit Edilemedi & 0.010 & $\mathrm{mg} / \mathrm{kg}$ & LC-MS/MS \\
\hline Metosulam & Tespit Edilemedi & 0.010 & $\mathrm{mg} / \mathrm{kg}$ & LC-MS/MS \\
\hline Metoxuron & Tespit Edilemedi & 0.010 & $\mathrm{mg} / \mathrm{kg}$ & LC-MS/MS \\
\hline Metrafenone & Tespit Edilemedi & 0.010 & $\mathrm{mg} / \mathrm{kg}$ & GC-MS \& GC-MS/MS \\
\hline Metribuzin * & Tespit Edilemedi & 0.010 & $\mathrm{mg} / \mathrm{kg}$ & LC-MS/MS \\
\hline Metsulfuron-methyl & Tespit Edilemedi & 0.010 & $\mathrm{mg} / \mathrm{kg}$ & LC-MS/MS \\
\hline Mevinphos * & Tespit Edilemedi & 0.010 & $\mathrm{mg} / \mathrm{kg}$ & GC-MS \& GC-MS/MS \\
\hline Mirex & Tespit Edilemedi & 0.010 & $\mathrm{mg} / \mathrm{kg}$ & GC-MS \& GC-MS/MS \\
\hline Molinate ${ }^{*}$ & Tespit Edilemedi & 0.010 & $\mathrm{mg} / \mathrm{kg}$ & GC-MS \& GC-MS/MS \\
\hline Monalide & Tespit Edilemedi & 0.010 & $\mathrm{mg} / \mathrm{kg}$ & GC-MS \& GC-MS/MS \\
\hline Monocrotophos & Tespit Edilemedi & 0.010 & $\mathrm{mg} / \mathrm{kg}$ & LC-MS/MS \\
\hline Monolinuron * & Tespit Edilemedi & 0.010 & $\mathrm{mg} / \mathrm{kg}$ & LC-MS/MS \\
\hline Monuron & Tespit Edilemedi & 0.010 & $\mathrm{mg} / \mathrm{kg}$ & LC-MS/MS \\
\hline Myclobutanil * & Tespit Edilemedi & 0.010 & $\mathrm{mg} / \mathrm{kg}$ & LC-MS/MS \\
\hline Naled ${ }^{*}$ & Tespit Edilemedi & 0.010 & $\mathrm{mg} / \mathrm{kg}$ & LC-MS/MS \\
\hline Napropamide & Tespit Edilemedi & 0.010 & $\mathrm{mg} / \mathrm{kg}$ & LC-MS/MS \\
\hline Neburon & Tespit Edilemedi & 0.010 & $\mathrm{mg} / \mathrm{kg}$ & LC-MS/MS \\
\hline Nicosulfuron & Tespit Edilemedi & 0.010 & $\mathrm{mg} / \mathrm{kg}$ & LC-MS/MS \\
\hline Nitenpyram & Tespit Edilemedi & 0.010 & $\mathrm{mg} / \mathrm{kg}$ & LC-MS/MS \\
\hline Nitralin & Tespit Edilemedi & 0.010 & $\mathrm{mg} / \mathrm{kg}$ & LC-MS/MS \\
\hline Nitrapyrin & Tespit Edilemedi & 0.010 & $\mathrm{mg} / \mathrm{kg}$ & GC-MS \& GC-MS/MS \\
\hline Nitrofen & Tespit Edilemedi & 0.010 & $\mathrm{mg} / \mathrm{kg}$ & GC-MS \& GC-MS/MS \\
\hline Nitrothal-Isopropyl & Tespit Edilemedi & 0.010 & $\mathrm{mg} / \mathrm{kg}$ & GC-MS \& GC-MS/MS \\
\hline Norflurazon & Tespit Edilemedi & 0.010 & $\mathrm{mg} / \mathrm{kg}$ & LC-MS/MS \\
\hline Novaluron & Tespit Edilemedi & 0.010 & $\mathrm{mg} / \mathrm{kg}$ & LC-MS/MS \\
\hline Nuarimol & Tespit Edilemedi & 0.010 & $\mathrm{mg} / \mathrm{kg}$ & LC-MS/MS \\
\hline Ofurace & Tespit Edilemedi & 0.010 & $\mathrm{mg} / \mathrm{kg}$ & LC-MS/MS \\
\hline Omethoate ${ }^{*}$ & Tespit Edilemedi & 0.010 & $\mathrm{mg} / \mathrm{kg}$ & LC-MS/MS \\
\hline Oxadiazon & Tespit Edilemedi & 0.010 & $\mathrm{mg} / \mathrm{kg}$ & GC-MS \& GC-MS/MS \\
\hline Oxadixyl ${ }^{*}$ & Tespit Edilemedi & 0.010 & $\mathrm{mg} / \mathrm{kg}$ & LC-MS/MS \\
\hline Oxamyl ${ }^{*}$ & Tespit Edilemedi & 0.010 & $\mathrm{mg} / \mathrm{kg}$ & LC-MS/MS \\
\hline Oxyfluorfen * & Tespit Edilemedi & 0.010 & $\mathrm{mg} / \mathrm{kg}$ & GC-MS \& GC-MS/MS \\
\hline Paclobutrazol & Tespit Edilemedi & 0.010 & $\mathrm{mg} / \mathrm{kg}$ & LC-MS/MS \\
\hline Paraoxon -ethyl & Tespit Edilemedi & 0.010 & $\mathrm{mg} / \mathrm{kg}$ & LC-MS/MS \\
\hline Paraoxon-methyl & Tespit Edilemedi & 0.010 & $\mathrm{mg} / \mathrm{kg}$ & LC-MS/MS \\
\hline Parathion-ethyl * & Tespit Edilemedi & 0.010 & $\mathrm{mg} / \mathrm{kg}$ & GC-MS \& GC-MS/MS \\
\hline Parathion-methyl ${ }^{*}$ & Tespit Edilemedi & 0.010 & $\mathrm{mg} / \mathrm{kg}$ & GC-MS \& GC-MS/MS \\
\hline Pebulat & Tespit Edilemedi & 0.010 & $\mathrm{mg} / \mathrm{kg}$ & LC-MS/MS \\
\hline Penconazole ${ }^{*}$ & Tespit Edilemedi & 0.010 & $\mathrm{mg} / \mathrm{kg}$ & LC-MS/MS \\
\hline Pencycuron & Tespit Edilemedi & 0.010 & $\mathrm{mg} / \mathrm{kg}$ & LC-MS/MS \\
\hline Pendimethalin * & Tespit Edilemedi & 0.010 & $\mathrm{mg} / \mathrm{kg}$ & LC-MS/MS \\
\hline Pentachloroaniline & Tespit Edilemedi & 0.010 & $\mathrm{mg} / \mathrm{kg}$ & GC-MS \& GC-MS/MS \\
\hline Pentachloroanisole & Tespit Edilemedi & 0.010 & $\mathrm{mg} / \mathrm{kg}$ & GC-MS \& GC-MS/MS \\
\hline Pentachlorobenzene & Tespit Edilemedi & 0.010 & $\mathrm{mg} / \mathrm{kg}$ & GC-MS \& GC-MS/MS \\
\hline
\end{tabular}




\begin{tabular}{|c|c|c|c|c|}
\hline Pentachlorophenol & Tespit Edilemedi & 0.010 & $\mathrm{mg} / \mathrm{kg}$ & GC-MS \& GC-MS/MS \\
\hline Pentanochlor * & Tespit Edilemedi & 0.010 & $\mathrm{mg} / \mathrm{kg}$ & GC-MS \& GC-MS/MS \\
\hline Permethrin * & Tespit Edilemedi & 0.010 & $\mathrm{mg} / \mathrm{kg}$ & GC-MS \& GC-MS/MS \\
\hline Perthane & Tespit Edilemedi & 0.010 & $\mathrm{mg} / \mathrm{kg}$ & GC-MS \& GC-MS/MS \\
\hline Pethoxamid * & Tespit Edilemedi & 0.010 & $\mathrm{mg} / \mathrm{kg}$ & GC-MS \& GC-MS/MS \\
\hline Phenkapton * & Tespit Edilemedi & 0.010 & $\mathrm{mg} / \mathrm{kg}$ & GC-MS \& GC-MS/MS \\
\hline Phenmedipham & Tespit Edilemedi & 0.010 & $\mathrm{mg} / \mathrm{kg}$ & LC-MS/MS \\
\hline Phenothrin * & Tespit Edilemedi & 0.010 & $\mathrm{mg} / \mathrm{kg}$ & GC-MS \& GC-MS/MS \\
\hline Phenthoate * & Tespit Edilemedi & 0.010 & $\mathrm{mg} / \mathrm{kg}$ & LC-MS/MS \\
\hline Phenylphenol, 2- (OPP) & Tespit Edilemedi & 0.010 & $\mathrm{mg} / \mathrm{kg}$ & GC-MS \& GC-MS/MS \\
\hline Phorate ${ }^{*}$ & Tespit Edilemedi & 0.010 & $\mathrm{mg} / \mathrm{kg}$ & GC-MS \& GC-MS/MS \\
\hline Phorate Sulfone * & Tespit Edilemedi & 0.010 & $\mathrm{mg} / \mathrm{kg}$ & LC-MS/MS \\
\hline Phorate Sulfoxide ${ }^{*}$ & Tespit Edilemedi & 0.010 & $\mathrm{mg} / \mathrm{kg}$ & LC-MS/MS \\
\hline Phosalone ${ }^{*}$ & Tespit Edilemedi & 0.010 & $\mathrm{mg} / \mathrm{kg}$ & LC-MS/MS \\
\hline Phosmet ${ }^{*}$ & Tespit Edilemedi & 0.010 & $\mathrm{mg} / \mathrm{kg}$ & LC-MS/MS \\
\hline Phosphamidon * & Tespit Edilemedi & 0.010 & $\mathrm{mg} / \mathrm{kg}$ & LC-MS/MS \\
\hline Phoxim * & Tespit Edilemedi & 0.010 & $\mathrm{mg} / \mathrm{kg}$ & LC-MS/MS \\
\hline Picloram * & Tespit Edilemedi & 0.010 & $\mathrm{mg} / \mathrm{kg}$ & LC-MS/MS \\
\hline Picolinafen & Tespit Edilemedi & 0.010 & $\mathrm{mg} / \mathrm{kg}$ & GC-MS \& GC-MS/MS \\
\hline Picoxystrobin & Tespit Edilemedi & 0.010 & $\mathrm{mg} / \mathrm{kg}$ & LC-MS/MS \\
\hline Pinoxaden & Tespit Edilemedi & 0.010 & $\mathrm{mg} / \mathrm{kg}$ & LC-MS/MS \\
\hline Piperonyl butoxide & Tespit Edilemedi & 0.010 & $\mathrm{mg} / \mathrm{kg}$ & LC-MS/MS \\
\hline Pirimicarb * & Tespit Edilemedi & 0.010 & $\mathrm{mg} / \mathrm{kg}$ & LC-MS/MS \\
\hline Pirimicarb, Desmethyl- & Tespit Edilemedi & 0.010 & $\mathrm{mg} / \mathrm{kg}$ & LC-MS/MS \\
\hline Pirimicarb, Desmethylformamido- & Tespit Edilemedi & 0.010 & $\mathrm{mg} / \mathrm{kg}$ & LC-MS/MS \\
\hline Pirimiphos-ethyl & Tespit Edilemedi & 0.010 & $\mathrm{mg} / \mathrm{kg}$ & GC-MS \& GC-MS/MS \\
\hline Pirimiphos-methyl ${ }^{*}$ & Tespit Edilemedi & 0.010 & $\mathrm{mg} / \mathrm{kg}$ & GC-MS \& GC-MS/MS \\
\hline Primsulfuron methyl ${ }^{*}$ & Tespit Edilemedi & 0.010 & $\mathrm{mg} / \mathrm{kg}$ & LC-MS/MS \\
\hline Prochloraz & Tespit Edilemedi & 0.010 & $\mathrm{mg} / \mathrm{kg}$ & LC-MS/MS \\
\hline Procymidone ${ }^{*}$ & Tespit Edilemedi & 0.010 & $\mathrm{mg} / \mathrm{kg}$ & GC-MS \& GC-MS/MS \\
\hline Profenofos ${ }^{*}$ & Tespit Edilemedi & 0.010 & $\mathrm{mg} / \mathrm{kg}$ & GC-MS \& GC-MS/MS \\
\hline Profluralin & Tespit Edilemedi & 0.010 & $\mathrm{mg} / \mathrm{kg}$ & GC-MS \& GC-MS/MS \\
\hline Profoxydim (Clefoxydim) & Tespit Edilemedi & 0.010 & $\mathrm{mg} / \mathrm{kg}$ & LC-MS/MS \\
\hline Promecarb & Tespit Edilemedi & 0.010 & $\mathrm{mg} / \mathrm{kg}$ & LC-MS/MS \\
\hline Prometon & Tespit Edilemedi & 0.010 & $\mathrm{mg} / \mathrm{kg}$ & GC-MS \& GC-MS/MS \\
\hline Prometryn * & Tespit Edilemedi & 0.010 & $\mathrm{mg} / \mathrm{kg}$ & LC-MS/MS \\
\hline Propachlor & Tespit Edilemedi & 0.010 & $\mathrm{mg} / \mathrm{kg}$ & LC-MS/MS \\
\hline Propamocarb * & Tespit Edilemedi & 0.010 & $\mathrm{mg} / \mathrm{kg}$ & LC-MS/MS \\
\hline Propanil & Tespit Edilemedi & 0.010 & $\mathrm{mg} / \mathrm{kg}$ & LC-MS/MS \\
\hline Propaquizafop * & Tespit Edilemedi & 0.010 & $\mathrm{mg} / \mathrm{kg}$ & LC-MS/MS \\
\hline Propargite * & Tespit Edilemedi & 0.010 & $\mathrm{mg} / \mathrm{kg}$ & LC-MS/MS \\
\hline Propazine * & Tespit Edilemedi & 0.010 & $\mathrm{mg} / \mathrm{kg}$ & LC-MS/MS \\
\hline Propetamphos & Tespit Edilemedi & 0.010 & $\mathrm{mg} / \mathrm{kg}$ & GC-MS \& GC-MS/MS \\
\hline Propham & Tespit Edilemedi & 0.010 & $\mathrm{mg} / \mathrm{kg}$ & LC-MS/MS \\
\hline Propiconazole * & Tespit Edilemedi & 0.010 & $\mathrm{mg} / \mathrm{kg}$ & LC-MS/MS \\
\hline Propoxur * & Tespit Edilemedi & 0.010 & $\mathrm{mg} / \mathrm{kg}$ & LC-MS/MS \\
\hline Propoxycarbazone & Tespit Edilemedi & 0.010 & $\mathrm{mg} / \mathrm{kg}$ & LC-MS/MS \\
\hline Propyzamide ${ }^{*}$ & Tespit Edilemedi & 0.010 & $\mathrm{mg} / \mathrm{kg}$ & LC-MS/MS \\
\hline Proquinazid & Tespit Edilemedi & 0.010 & $\mathrm{mg} / \mathrm{kg}$ & LC-MS/MS \\
\hline Prosulfocarb & Tespit Edilemedi & 0.010 & $\mathrm{mg} / \mathrm{kg}$ & LC-MS/MS \\
\hline Prosulfuron & Tespit Edilemedi & 0.010 & $\mathrm{mg} / \mathrm{kg}$ & LC-MS/MS \\
\hline Prothioconazole & Tespit Edilemedi & 0.010 & $\mathrm{mg} / \mathrm{kg}$ & LC-MS/MS \\
\hline Prothioconazole-desthio ${ }^{*}$ & Tespit Edilemedi & 0.010 & $\mathrm{mg} / \mathrm{kg}$ & LC-MS/MS \\
\hline Prothiofos * & Tespit Edilemedi & 0.010 & $\mathrm{mg} / \mathrm{kg}$ & GC-MS \& GC-MS/MS \\
\hline Pymetrozine & Tespit Edilemedi & 0.010 & $\mathrm{mg} / \mathrm{kg}$ & LC-MS/MS \\
\hline Pyraclostrobin & Tespit Edilemedi & 0.010 & $\mathrm{mg} / \mathrm{kg}$ & LC-MS/MS \\
\hline Pyraflufen-ethyl & Tespit Edilemedi & 0.010 & $\mathrm{mg} / \mathrm{kg}$ & GC-MS \& GC-MS/MS \\
\hline Pyrazophos * & Tespit Edilemedi & 0.010 & $\mathrm{mg} / \mathrm{kg}$ & GC-MS \& GC-MS/MS \\
\hline Pyrethrins 1 & Tespit Edilemedi & 0.010 & $\mathrm{mg} / \mathrm{kg}$ & LC-MS/MS \\
\hline Pyrethrins 2 & Tespit Edilemedi & 0.010 & $\mathrm{mg} / \mathrm{kg}$ & LC-MS/MS \\
\hline Pyridaben * & Tespit Edilemedi & 0.010 & $\mathrm{mg} / \mathrm{kg}$ & LC-MS/MS \\
\hline Pyridalyl * & Tespit Edilemedi & 0.010 & $\mathrm{mg} / \mathrm{kg}$ & GC-MS \& GC-MS/MS \\
\hline
\end{tabular}




\begin{tabular}{|c|c|c|c|c|}
\hline Pyridaphenthion * & Tespit Edilemedi & 0.010 & $\mathrm{mg} / \mathrm{kg}$ & LC-MS/MS \\
\hline Pyridate ${ }^{*}$ & Tespit Edilemedi & 0.010 & $\mathrm{mg} / \mathrm{kg}$ & LC-MS/MS \\
\hline Pyrifenox & Tespit Edilemedi & 0.010 & $\mathrm{mg} / \mathrm{kg}$ & LC-MS/MS \\
\hline Pyrimethanil * & Tespit Edilemedi & 0.010 & $\mathrm{mg} / \mathrm{kg}$ & GC-MS \& GC-MS/MS \\
\hline Pyrimidifen & Tespit Edilemedi & 0.010 & $\mathrm{mg} / \mathrm{kg}$ & GC-MS \& GC-MS/MS \\
\hline Pyriproxyfen * & Tespit Edilemedi & 0.010 & $\mathrm{mg} / \mathrm{kg}$ & LC-MS/MS \\
\hline Quinalphos * & Tespit Edilemedi & 0.010 & $\mathrm{mg} / \mathrm{kg}$ & GC-MS \& GC-MS/MS \\
\hline Quinmerac * & Tespit Edilemedi & 0.010 & $\mathrm{mg} / \mathrm{kg}$ & LC-MS/MS \\
\hline Quinoclamine & Tespit Edilemedi & 0.010 & $\mathrm{mg} / \mathrm{kg}$ & LC-MS/MS \\
\hline Quinoxyfen * & Tespit Edilemedi & 0.010 & $\mathrm{mg} / \mathrm{kg}$ & GC-MS \& GC-MS/MS \\
\hline Quintozene* & Tespit Edilemedi & 0.010 & $\mathrm{mg} / \mathrm{kg}$ & GC-MS \& GC-MS/MS \\
\hline Quizalafop-p-ethyl * & Tespit Edilemedi & 0.010 & $\mathrm{mg} / \mathrm{kg}$ & LC-MS/MS \\
\hline Quizalofop free acid & Tespit Edilemedi & 0.010 & $\mathrm{mg} / \mathrm{kg}$ & LC-MS/MS \\
\hline Quizalofop-ethyl & Tespit Edilemedi & 0.010 & $\mathrm{mg} / \mathrm{kg}$ & GC-MS \& GC-MS/MS \\
\hline Rabenzazole & Tespit Edilemedi & 0.010 & $\mathrm{mg} / \mathrm{kg}$ & LC-MS/MS \\
\hline Rimsulfuron & Tespit Edilemedi & 0.010 & $\mathrm{mg} / \mathrm{kg}$ & LC-MS/MS \\
\hline Rotenone & Tespit Edilemedi & 0.010 & $\mathrm{mg} / \mathrm{kg}$ & LC-MS/MS \\
\hline $\mathrm{S} 421$ & Tespit Edilemedi & 0.010 & $\mathrm{mg} / \mathrm{kg}$ & GC-MS \& GC-MS/MS \\
\hline Secbumeton & Tespit Edilemedi & 0.010 & $\mathrm{mg} / \mathrm{kg}$ & LC-MS/MS \\
\hline Sethoxydim & Tespit Edilemedi & 0.010 & $\mathrm{mg} / \mathrm{kg}$ & LC-MS/MS \\
\hline Silafluofen & Tespit Edilemedi & 0.010 & $\mathrm{mg} / \mathrm{kg}$ & GC-MS \& GC-MS/MS \\
\hline Silthiofam & Tespit Edilemedi & 0.010 & $\mathrm{mg} / \mathrm{kg}$ & LC-MS/MS \\
\hline Simazine ${ }^{*}$ & Tespit Edilemedi & 0.010 & $\mathrm{mg} / \mathrm{kg}$ & LC-MS/MS \\
\hline Simetryn & Tespit Edilemedi & 0.010 & $\mathrm{mg} / \mathrm{kg}$ & LC-MS/MS \\
\hline Spinosad * & Tespit Edilemedi & 0.010 & $\mathrm{mg} / \mathrm{kg}$ & LC-MS/MS \\
\hline Spinosad A * & Tespit Edilemedi & 0.010 & $\mathrm{mg} / \mathrm{kg}$ & LC-MS/MS \\
\hline Spinosad D * & Tespit Edilemedi & 0.010 & $\mathrm{mg} / \mathrm{kg}$ & LC-MS/MS \\
\hline Spirodiclofen & Tespit Edilemedi & 0.010 & $\mathrm{mg} / \mathrm{kg}$ & LC-MS/MS \\
\hline Spiromesifen & Tespit Edilemedi & 0.010 & $\mathrm{mg} / \mathrm{kg}$ & GC-MS \& GC-MS/MS \\
\hline $\begin{array}{l}\text { Spirotetramat and its } 4 \\
\text { metabolites BYI08330-enol, } \\
\text { BYI08330-ketohydroxy, } \\
\text { BYI08330-monohydroxy, and } \\
\text { BYI08330 enol-glucoside, } \\
\text { expressed as spirotetramat * }\end{array}$ & Tespit Edilemedi & 0.010 & $\mathrm{mg} / \mathrm{kg}$ & LC-MS/MS \\
\hline Spirotetramat Enol ${ }^{*}$ & Tespit Edilemedi & 0.010 & $\mathrm{mg} / \mathrm{kg}$ & LC-MS/MS \\
\hline Spirotetramat Enol Glucoside ${ }^{*}$ & Tespit Edilemedi & 0.010 & $\mathrm{mg} / \mathrm{kg}$ & LC-MS/MS \\
\hline Spirotetramat Keto-hydroxy ${ }^{*}$ & Tespit Edilemedi & 0.010 & $\mathrm{mg} / \mathrm{kg}$ & LC-MS/MS \\
\hline Spirotetramat Monohydroxy * & Tespit Edilemedi & 0.010 & $\mathrm{mg} / \mathrm{kg}$ & LC-MS/MS \\
\hline Spiroxamine * & Tespit Edilemedi & 0.010 & $\mathrm{mg} / \mathrm{kg}$ & LC-MS/MS \\
\hline Sulcotrione * & Tespit Edilemedi & 0.010 & $\mathrm{mg} / \mathrm{kg}$ & LC-MS/MS \\
\hline Sulfentrazone & Tespit Edilemedi & 0.010 & $\mathrm{mg} / \mathrm{kg}$ & LC-MS/MS \\
\hline Sulfotep & Tespit Edilemedi & 0.010 & $\mathrm{mg} / \mathrm{kg}$ & GC-MS \& GC-MS/MS \\
\hline Sulprofos & Tespit Edilemedi & 0.010 & $\mathrm{mg} / \mathrm{kg}$ & GC-MS \& GC-MS/MS \\
\hline Tau-fluvalinate (Fluvalinate) * & Tespit Edilemedi & 0.010 & $\mathrm{mg} / \mathrm{kg}$ & GC-MS \& GC-MS/MS \\
\hline Tebuconazole ${ }^{*}$ & Tespit Edilemedi & 0.010 & $\mathrm{mg} / \mathrm{kg}$ & LC-MS/MS \\
\hline Tebufenozide & Tespit Edilemedi & 0.010 & $\mathrm{mg} / \mathrm{kg}$ & LC-MS/MS \\
\hline Tebufenpyrad & Tespit Edilemedi & 0.010 & $\mathrm{mg} / \mathrm{kg}$ & LC-MS/MS \\
\hline Tebutam & Tespit Edilemedi & 0.010 & $\mathrm{mg} / \mathrm{kg}$ & LC-MS/MS \\
\hline Tecnazene * & Tespit Edilemedi & 0.010 & $\mathrm{mg} / \mathrm{kg}$ & GC-MS \& GC-MS/MS \\
\hline Teflubenzuron & Tespit Edilemedi & 0.010 & $\mathrm{mg} / \mathrm{kg}$ & LC-MS/MS \\
\hline Tefluthrin & Tespit Edilemedi & 0.010 & $\mathrm{mg} / \mathrm{kg}$ & GC-MS \& GC-MS/MS \\
\hline Tepraloxydim * & Tespit Edilemedi & 0.010 & $\mathrm{mg} / \mathrm{kg}$ & LC-MS/MS \\
\hline Terbacil & Tespit Edilemedi & 0.010 & $\mathrm{mg} / \mathrm{kg}$ & LC-MS/MS \\
\hline Terbufos & Tespit Edilemedi & 0.010 & $\mathrm{mg} / \mathrm{kg}$ & LC-MS/MS \\
\hline Terbumeton & Tespit Edilemedi & 0.010 & $\mathrm{mg} / \mathrm{kg}$ & LC-MS/MS \\
\hline Terbuthylazine * & Tespit Edilemedi & 0.010 & $\mathrm{mg} / \mathrm{kg}$ & GC-MS \& GC-MS/MS \\
\hline Terbutryn * & Tespit Edilemedi & 0.010 & $\mathrm{mg} / \mathrm{kg}$ & LC-MS/MS \\
\hline Tetrachlorvinphos * & Tespit Edilemedi & 0.010 & $\mathrm{mg} / \mathrm{kg}$ & GC-MS \& GC-MS/MS \\
\hline Tetraconazole & Tespit Edilemedi & 0.010 & $\mathrm{mg} / \mathrm{kg}$ & LC-MS/MS \\
\hline Tetradifon * & Tespit Edilemedi & 0.010 & $\mathrm{mg} / \mathrm{kg}$ & GC-MS \& GC-MS/MS \\
\hline Tetramethrin & Tespit Edilemedi & 0.010 & $\mathrm{mg} / \mathrm{kg}$ & GC-MS \& GC-MS/MS \\
\hline Tetrasul & Tespit Edilemedi & 0.010 & $\mathrm{mg} / \mathrm{kg}$ & GC-MS \& GC-MS/MS \\
\hline
\end{tabular}




\begin{tabular}{|c|c|c|c|c|}
\hline Thiabendazole * & Tespit Edilemedi & 0.010 & $\mathrm{mg} / \mathrm{kg}$ & LC-MS/MS \\
\hline Thiacloprid * & Tespit Edilemedi & 0.010 & $\mathrm{mg} / \mathrm{kg}$ & LC-MS/MS \\
\hline Thiamethoxam * & Tespit Edilemedi & 0.010 & $\mathrm{mg} / \mathrm{kg}$ & LC-MS/MS \\
\hline Thifensulfuron-methyl & Tespit Edilemedi & 0.010 & $\mathrm{mg} / \mathrm{kg}$ & LC-MS/MS \\
\hline Thiobencarb (Benthiocarb) & Tespit Edilemedi & 0.010 & $\mathrm{mg} / \mathrm{kg}$ & LC-MS/MS \\
\hline Thiodicarb & Tespit Edilemedi & 0.010 & $\mathrm{mg} / \mathrm{kg}$ & LC-MS/MS \\
\hline Thiofanox & Tespit Edilemedi & 0.010 & $\mathrm{mg} / \mathrm{kg}$ & LC-MS/MS \\
\hline Thiofanox-sulfone & Tespit Edilemedi & 0.010 & $\mathrm{mg} / \mathrm{kg}$ & LC-MS/MS \\
\hline Thiofanox-sulfoxide & Tespit Edilemedi & 0.010 & $\mathrm{mg} / \mathrm{kg}$ & LC-MS/MS \\
\hline Thiometon & Tespit Edilemedi & 0.010 & $\mathrm{mg} / \mathrm{kg}$ & GC-MS \& GC-MS/MS \\
\hline Thionazin & Tespit Edilemedi & 0.010 & $\mathrm{mg} / \mathrm{kg}$ & LC-MS/MS \\
\hline Thiophanate-methyl * & Tespit Edilemedi & 0.010 & $\mathrm{mg} / \mathrm{kg}$ & LC-MS/MS \\
\hline Tolclofos-methyl ${ }^{*}$ & Tespit Edilemedi & 0.010 & $\mathrm{mg} / \mathrm{kg}$ & GC-MS \& GC-MS/MS \\
\hline Tolfenpyrad & Tespit Edilemedi & 0.010 & $\mathrm{mg} / \mathrm{kg}$ & LC-MS/MS \\
\hline Tolylfluanid * & Tespit Edilemedi & 0.010 & $\mathrm{mg} / \mathrm{kg}$ & GC-MS \& GC-MS/MS \\
\hline Topramezone & Tespit Edilemedi & 0.010 & $\mathrm{mg} / \mathrm{kg}$ & LC-MS/MS \\
\hline Tralkoxydim & Tespit Edilemedi & 0.010 & $\mathrm{mg} / \mathrm{kg}$ & LC-MS/MS \\
\hline Transfluthrin & Tespit Edilemedi & 0.010 & $\mathrm{mg} / \mathrm{kg}$ & GC-MS \& GC-MS/MS \\
\hline Triadimefon * & Tespit Edilemedi & 0.010 & $\mathrm{mg} / \mathrm{kg}$ & LC-MS/MS \\
\hline Triadimenol * & Tespit Edilemedi & 0.010 & $\mathrm{mg} / \mathrm{kg}$ & LC-MS/MS \\
\hline Tri-allate ${ }^{*}$ & Tespit Edilemedi & 0.010 & $\mathrm{mg} / \mathrm{kg}$ & LC-MS/MS \\
\hline Triamiphos & Tespit Edilemedi & 0.010 & $\mathrm{mg} / \mathrm{kg}$ & LC-MS/MS \\
\hline Triasulfuron & Tespit Edilemedi & 0.010 & $\mathrm{mg} / \mathrm{kg}$ & LC-MS/MS \\
\hline Triazamat & Tespit Edilemedi & 0.010 & $\mathrm{mg} / \mathrm{kg}$ & LC-MS/MS \\
\hline Triazophos * & Tespit Edilemedi & 0.010 & $\mathrm{mg} / \mathrm{kg}$ & GC-MS \& GC-MS/MS \\
\hline Trichlorfon * & Tespit Edilemedi & 0.010 & $\mathrm{mg} / \mathrm{kg}$ & LC-MS/MS \\
\hline Trichloronat & Tespit Edilemedi & 0.010 & $\mathrm{mg} / \mathrm{kg}$ & GC-MS \& GC-MS/MS \\
\hline Triclopyr & Tespit Edilemedi & 0.010 & $\mathrm{mg} / \mathrm{kg}$ & LC-MS/MS \\
\hline Tricyclazole & Tespit Edilemedi & 0.010 & $\mathrm{mg} / \mathrm{kg}$ & LC-MS/MS \\
\hline Tridemorph & Tespit Edilemedi & 0.010 & $\mathrm{mg} / \mathrm{kg}$ & LC-MS/MS \\
\hline Trifloxystrobin * & Tespit Edilemedi & 0.010 & $\mathrm{mg} / \mathrm{kg}$ & LC-MS/MS \\
\hline Triflumizole * & Tespit Edilemedi & 0.010 & $\mathrm{mg} / \mathrm{kg}$ & LC-MS/MS \\
\hline Triflumuron & Tespit Edilemedi & 0.010 & $\mathrm{mg} / \mathrm{kg}$ & LC-MS/MS \\
\hline Trifluralin * & Tespit Edilemedi & 0.010 & $\mathrm{mg} / \mathrm{kg}$ & GC-MS \& GC-MS/MS \\
\hline Triflusulfuron-methyl & Tespit Edilemedi & 0.010 & $\mathrm{mg} / \mathrm{kg}$ & LC-MS/MS \\
\hline Triforine & Tespit Edilemedi & 0.010 & $\mathrm{mg} / \mathrm{kg}$ & LC-MS/MS \\
\hline Trimethacarb, 3,4,5-(Landrin) & Tespit Edilemedi & 0.010 & $\mathrm{mg} / \mathrm{kg}$ & LC-MS/MS \\
\hline Triticonazole & Tespit Edilemedi & 0.010 & $\mathrm{mg} / \mathrm{kg}$ & LC-MS/MS \\
\hline Uniconazole & Tespit Edilemedi & 0.010 & $\mathrm{mg} / \mathrm{kg}$ & LC-MS/MS \\
\hline Vamidothion * & Tespit Edilemedi & 0.010 & $\mathrm{mg} / \mathrm{kg}$ & LC-MS/MS \\
\hline Vinclozolin * & Tespit Edilemedi & 0.010 & $\mathrm{mg} / \mathrm{kg}$ & GC-MS \& GC-MS/MS \\
\hline Zoxamide & Tespit Edilemedi & 0.010 & $\mathrm{mg} / \mathrm{kg}$ & LC-MS/MS \\
\hline
\end{tabular}

*:Bu analizler akreditasyon kapsamındadır. (TÜRKAK'tan AB-0328-T ile TS EN ISO/IEC 17025:2012 standardına göre akreditedir.)

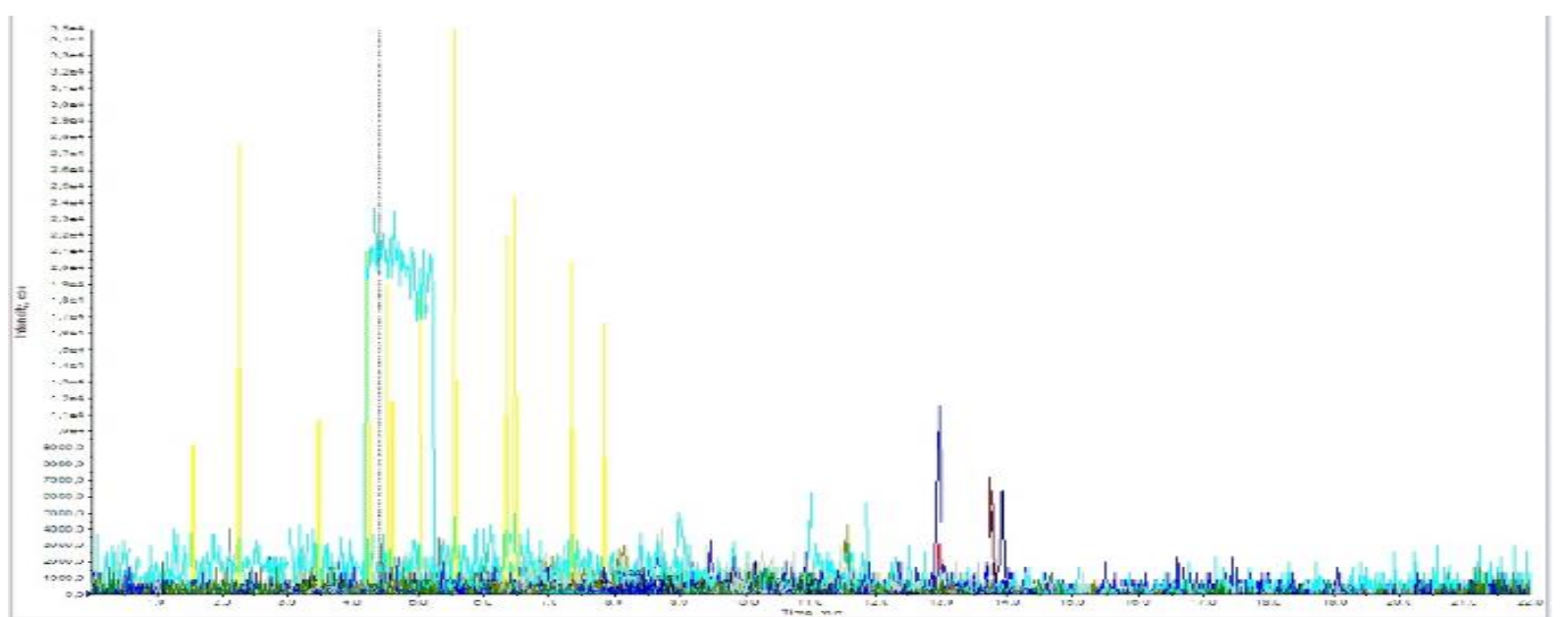

Şekil 1. Kaçak çaylardan elde edilen LC-MS/MS kromatogramı

Analiz sonucunda herhangi bir pestisit kalıntısına rastlanılmamış olup, elde edilen mevcut renkli piklerin ise; pestisit ifade etmediği, cihaz ve ortam kaynaklı gürültü pikleri olduğu belirlenmiştir. 


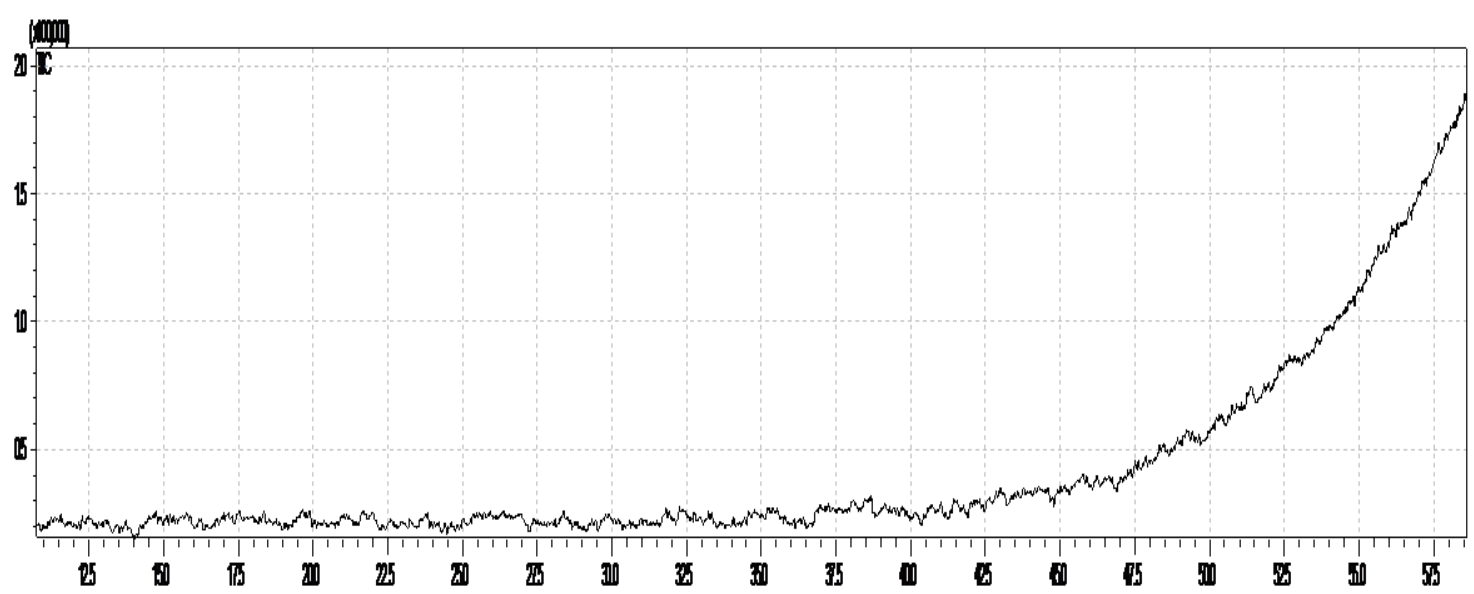

Şekil 2. Kaçak çaylardan elde edilen GC-MS kromatogramı

Analiz sonucunda herhangi bir pestisit kalıntısına rastlanılmamış olup, elde edilen mevcut devam eden pik görüntüleri ise; pestisit ifade etmediği, cihaz ve ortam kaynaklı gürültü pikleri olduğu belirlenmiştir.

Mevcut araştırmada analizi yapılan bileşikler, tarımda en yaygın kullanılan ve içerisinden insan sağlı̆̆ına zararlı olduğu iddia edilen 594 pestisit etken maddesine göre seçilmiştir. Çay bitkisini böceklerden korumak amacıyla pestisit ilaçları (Deltamethrin, Dimethoate, Dicofol vb.) kullanılmaktadır. Her iki faktöründe sağllk üzerindeki olumsuz etkileri yapılan pek çok deneyle ortaya konulmuştur (Soyöz, 2003). Pestisit analizi için yapılan LC-MS ve GC-MS analizleri molekül ağırlığına duyarlı olarak kurgulandığı için oldukça hassastır. Türkiye'de tüketilen kaçak çaylarda pestisit analizi ile ilgili daha önce hiçbir çalışma yapılmamış olması, çalışmamızı oldukça özgün kılmaktadır ve elde ettiğimiz sonuçlar dikkat çekicidir.

İran çayının üretildiği Hazar Denizi kıyısındaki nemli iklim sebebiyle çay bitkisinde aşırı böceklenme meydana geldiği bildirilmiştir. Böceklenme ile mücadele edilmesi için kimyasal (pestisit) ilaçlar kullanılmaktadır. İran'da üretilen çaylarda bu sebepten dolayı yüksek miktarda böcek ve kimyasal (pestisit) ilaç kalıntıları bulunduğu iddia edilmiştir. (Anonim 2018-B). Ancak yaptığımız analizlerde kaçak çayların herhangi bir pestisit içerdiğine dair bulguya rastlanmamıştır. Bu beklenmedik sonucu teyit etmek üzere analiz şirketi değiştirilerek ve numune sayısı artırılarak tekrar analiz yapılmıştır. Ancak bu çalışmada da herhangi bir pestisit kalıntısına rastlanmamıştır. Bu sonuçlar ise; İran ve Sri Lanka menşeli kaçak çay hakkında iddia edilenlerin aksine olarak bulunmuştur. Çalışma alan itibarıyla dar bir bölgeyi kapsıyor olsa da kaçak çay hakkında verilen peşin hükümleri ve ön yargıları değiştirecek niteliktedir. Bu konu hakkında yapılacak daha geniş alanlı ve detaylı çalışmalara kadar bu iddialar spekülasyon olarak kalmaya devam edecektir. Güney Doğu Anadolu Bölgesinde yaygın olarak kullanılan kaçak çayların pestisit içerip içermediği ile ilgili net bir hüküm verebilmek için tüm bölgeyi kapsayan daha geniş araştırmaların yapılması gerekmektedir.

\section{KAYNAKLAR}

Anonim 2018a. http://www.turkcayi.com/html/ OrganikCayBirizBizden.html (Erişim Tarihi: $01.02 .2018)$

Anonim 2018b. http://ulusalcaykonseyi.org.tr///201301-29-17-56-42/cay-sektoerue-sorunlar-vecoezuemoenerileri, (Erişim Tarihi: 22.02.2018)

Anonim 2018c. https://m.haberler.com/diyarbakir-dadogal-ve-saglikli-caykur-sempozyumu-7237772haberi/' (Erişim Tarihi: 01.02.2018)

Er B 2013. Çay Tarımında Yaşanan Sorunların Çözümünde Alternatif Ürün Likapa. II. Rize Kalkınma Sempozyumu, 3 -4 Mayıs 2013, Rize.

FAO 2013. FAO web page, http://faostat.fao.org/site/ (23/04/2013).

Gültekin S 2013. Organik Tarım Kümelenmesi ve Çay Sektörünün Bölgesel Rekabet Analizi. II. Rize Kalkınma Sempozyumu, 3-4 Mayıs 2013, Rize.

Harman C 2014. Karadeniz Bölge'sinde Endemik Tarım Ürünleri: Fındık, Çay ve Kivi'nin Üretimi Pazarlanması ve Tüketimi. Giresun Üniversitesi Sos Bil Ens., İktisat A.B.D Yüksek Lisans Tezi, $145 \mathrm{~s}$

Kaya E, Tüzmen MN, Yücel H. Yücel N, Demiryas N 2006. Oksidatif Doku Hasarında Bazı Organofosfatlı Pestisidlerin Rolleri." XX. Ulusal Kimya Kongresi, Erciyes Üniversitesi, 4-8 Eylül, Kayseri.

Kurt G, Hacıŏlu HK 2013. Dünya Ülkeleri ile Türkiye'nin Çay Üretiminin İstatistiklerle İncelenmesi. II. Rize Kalkınma Sempozyumu, 3-4 Mayıs 2013 Rize.

Lesueur C, Knittl P, Gartner M, Mentler A, Fuerhacker M. Analysis of 140 pesticides from conventional farming foodstuff samples after 
extraction with the modified QuECheRS method. Food Control. 19(9):906-14.

Özcan M, Yazıcıŏlu E 2013. Türkiye Çay Yetiştiriciliğinin Sorunları Ve Öncelikleri. II. Rize Kalkınma Sempozyumu, 3-4 Mayıs 2013, Rize.

Rize Ticaret Borsası, 2014. Çay Sektörü Sorunlar ve Çözüm Önerileri”, Rize.

Soyöz M, Özçelik N 2003. Zirai mücadelede kullanılan pestisitlerin sitogenetik etkileri. SDÜ Tıp Fakültesi Dergisi, 10 (1) : 6-9.

Şensoy RİG, Ersayar L, Doğan A 2017. Van İlinde Satılmakta Olan Yaş ve Kuru Üzümler İle
Salamura Asma Yapraklarında Pestisit Kalıntı Miktarlarının Belirlenmesi. Yüzüncü Y Yl Üniversitesi Tarım Bilimleri Dergisi, 27 (3): 436446.

Üstün Ç, Demirci N 2013. Çay Bitkisinin (Camellia Sınensıs L.) Tarihsel Gelişimi ve Tıbbi Açıdan Değerlendirilmesi. Lokman Hekim Journal, 3(3): 512

Zenginbal H 2013. "Rize İli Bahçe Bitkileri Yetiştiriciliğinde Çay Tarımının Yeri, Sorunları ve Çözüm Önerileri. II. Rize Kalkınma Sempozyumu, 3-4 Mayıs 2013 Rize. 